\title{
DIPLOMATEN ALS PATRONE UND KLIENTEN: DER EINFLUSS PERSONALER VERFLECHTUNGEN IN DER FRANZÖSISCHEN DIPLOMATIE AUF DEM WESTFÄLISCHEN FRIEDENSKONGRESS
}

Von "Diplomaten als Patronen und Klienten« im Frankreich des 17. Jahrhunderts zu reden, bedarf in verschiedener Hinsicht einer begrifflichen Erläute-

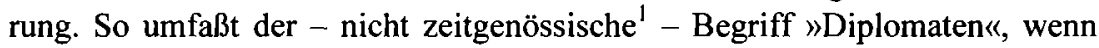
man ihn für französische Repräsentanten im 17. Jahrhundert verwendet, sehr unterschiedliche Personenkreise: hochrangige ambassadeurs (Botschafter) ${ }^{2}$, nicht näher spezifizierte Gesandte und niedrigrangige Vertreter, worunter alle vom Residenten abwärts zu verstehen sind. Botschafter wurde nur, wer strikte gesellschaftliche und finanzielle Voraussetzungen erfüllte ${ }^{3}$. Botschafter hätten

1 Zur erstmaligen Verwendung der Begriffe »diplomate « und »diplomatie« in der französischen und nachfolgend in der deutschen Sprache siehe: Trésor de la Langue Française, Bd. VII, hg. v. Paul IMBS, Paris 1979, S. 236f.; Nouveau dictionnaire étymologique et historique, hg. v. Albert DAUZAT, Jean DUBOIS, Henri MITTERAND, Paris 1964, S. 237; Friedrich KLUGE, Etymologisches Wörterbuch der deutschen Sprache, Berlin ${ }^{21} 1975$, S. 134.

2 Das Frühneuhochdeutsche kennt keinen adäquaten Begriff. Verwendet wird das französische »ambassadeur« neben dem ebenfalls geläufigen lateinischen »legatus«; zur Gleichsetzung und zur Bedeutungsdifferenz beider siehe Jean HOTMAN DE VILLIERS, De la Charge et Dignité de l'Ambassadeur, Paris ${ }^{2} 1604$ [zitiertes Exemplar: Bibliothèque Nationale de France (BNF) *E 2338 (1)], unfoliiertes Vorwort; Garrett MATTINGLY, Renaissance Diplomacy, Boston 1955, S. 25. - Im folgenden wird der Begriff »Botschafter« synonym für »ambassadeur« verwendet.

3 Zum diplomatischen Corps Mazarins nach 1648, insbesondere auch zur sozialen Herkunft der Diplomaten, siehe Dennis H. O'BRIEN, Mazarin's Diplomatic Corps, 1648-166I. In: North Dakota Quarterly 45 (1977) S. 31-42. Die verschiedenen Bedingungen für eine Botschafter-Ernennung in der Epoche Ludwigs XIV. analysiert William James RoOSEN, The True Ambassador: Occupational and Personal Characteristics of French Ambassadors under Louis XIV. In: European Studies Review 3 (1973) S. 121-139; DERS., The Age of Louis XIV. The Rise of Modern Diplomacy, Cambridge (Mass.) 1976, S. $60 \mathrm{ff}$. Grundsätzliche Aussagen dazu von Mazarin selbst finden sich in einem Schreiben an die französischen Botschafter in Münster von 1644; Acta Pacis Westphalicae (APW), hg. v. Max BRAUBACH und Konrad REPGEN, Münster, Serie II B (Französische Korrespondenz), Bd. 1, bearb. v. Ursula IRSIGLER, 1979, nr. 46 S. 86. Siehe darüber hinaus den Artikel »Ambassadeurs« von Jean BÉRENGER in: Lucien BÉLY (Hg.), Dictionnaire de l'Ancien Régime, Paris 1996, S. 54f.; zur diplomatischen Hierarchie siehe Lucien BÉLY, Espions et ambassadeurs au temps de Louis XIV, Paris 1990, S. 291ff.; C[amille]- 
sich schon deshalb kaum als eine Einheit mit niedrigrangigen Diplomaten gesehen. Eine Ernennung zum Botschafter war zudem - je nach Bedeutung der Mission - Ausdruck einer gehobenen politischen Stellung oder sollte der Weg hin zu einer solchen sein. So saßen von den drei französischen Botschaftern auf dem Westfälischen Friedenskongreß zwei - der Herzog von Longueville und der Graf d'Avaux - im Conseil d'en haut, dem höchsten Regierungsrat; der dritte, Abel Servien, wurde nach Abberufung seiner Kollegen im April 1648 dorthin berufen ${ }^{4}$. Botschafter waren oft weniger eine Einheit mit niedrigeren Diplomaten denn mit der höfischen Regierung und den dortigen Politi$\mathrm{kern}^{5}$. Personale Verflechtungen in der Diplomatie waren deshalb kein Sonderfall: Diplomaten waren fördernde Patrone oder geförderte Klienten im großen Rahmen der allgemeinen politischen Personenbeziehungen, die dem politischen Aufstieg und der Erlangung und Sicherung von Macht dienten ${ }^{6}$.

Personale Verflechtungen « oder »Netzwerke« im Dienst des gesellschaftlichen oder politischen Aufstiegs sind durch Wolfgang Reinhard zu einem zentralen Forschungsthema geworden ${ }^{7}$. Vor ihm haben Roland Mousnier ${ }^{8}$ und Orest Ranum ${ }^{9}$ die spezielle Bedeutung von Klientelbeziehungen in der französischen Regierung herausgearbeitet. Erst Reinhard beleuchtete jedoch die all-

G[eorges] PICAVET, La "Carrière« diplomatique en France au temps de Louis XIV (1661-1715), in: Revue d'Histoire économique et sociale 11 (1923) S. 383-408.

4 Longueville und d'Avaux hatten seit 1643 einen Sitz im Conseil. Longueville hatte ihn anläßlich seiner Ernennung zum Botschafter erhalten, als Kompensation für das - für ihn als einflußreichen Hochadeligen - wenig attraktive Amt, aber auch, um der Mission den entsprechenden Rückhalt zu geben. Er ging auf diese Umstände der Ernennung in einem Schreiben an Mazarin vom 30. Juli 1646 ein; Ausfertigung: Archives du Ministère des Affaires étrangères Paris (AE), Série Correspondance Politique (CP) Allemagne 61 fol. 198-204'. Servien hatte bereits als Staatssekretär unter Richelieu an ConseilSitzungen teilgenommen, ohne einen festen Sitz zu haben. Er wurde am 24. April 1648 durch Mazarin von der am Vortag erfolgten Berufung informiert; Ausfertigung des Schreibens: AE, CP ALLEMAGNE 119 fol. 391.

5 Die französischen Botschafter des Westfälischen Friedenskongresses berieten und handelten deutlich über den Rahmen des eigentlichen Kongreßgeschehens hinaus: 1644 fertigten sie die Instruktion für eine Gesandtschaft nach Siebenbürgen an, 1645 wurden sie mit der für eine - nicht verwirklichte - Botschafter-Mission nach Schweden beauftragt. Umstritten war zwischen d'Avaux und Servien allerdings, ob der weite Handlungsrahmen eine besondere Gnade sei oder, wie es das Conseil-Mitglied d'Avaux sah, sich bereits aus den eigenen Ämtern herleite. Dazu die Schreiben Serviens an Lionne vom 15. sowie Mazarins an d'Avaux und Servien vom 28. Januar 1645; APW II B (wie Anm. 3), Bd. 2, bearb. v. Franz BosBACH, 1986, nr. 14 S. 63 f. und nr. 24 S. 88.

6 Vgl. dazu auch Rainer BABEL, Einleitung, in: DERS. (Hg.), Frankreich im europäischen Staatensystem der Frühen Neuzeit (Beihefte der Francia, 35), Sigmaringen 1995, S. 7-11, hier S. 8.

7 Wolfgang REINHARD, Freunde und Kreaturen. »Verflechtung« als Konzept zur Erforschung historischer Führungsgruppen. Römische Oligarchie um 1600, München 1979.

8 Roland MOUSNIER, Les Hiérarchies sociales de 1450 à nos jours, Paris 1969.

9 Orest A. RANUM, Richelieu and the Councillors of Louis XIII, Oxford 1963. 
gemeine Struktur von herrschaftsbildenden und herrschaftsstabilisierenden Verflechtungen, die über bloße Klientelsysteme hinausgehen. Netzwerke sind, wie Reinhard herausarbeitete, komplexer als Klientelsysteme, weil Menschen durch zahlreiche Faktoren über den der Patronage hinaus verbunden sein können: Freundschaft, gleiche Herkunft, gemeinsame Weltanschauung, finanzielle Abhängigkeit ${ }^{10}$ etc. Es ist insofern zu bedauern, daß Sharon Kettering, die als erste systematisch Personenbeziehungen als Element der Herrschaftsausübung in Frankreich im 17. Jahrhundert untersuchte, sich dabei weitgehend auf Klientelbeziehungen beschränkte ", die allerdings - anders als andere personale Verflechtungen - eine strukturelle Vorstufe des Regierungssystems Ludwigs XIV. bildeten ${ }^{12}$ und unter die sich politisch relevante Beziehungen in Frankreich - gerade auch für den Fall der Diplomaten auf dem Westfälischen Friedenskongre $\beta^{13}$ - subsumieren lassen, wenn auch Verwandte und Freunde bevorzugt als Klienten rekrutiert wurden ${ }^{14}$.

${ }^{10}$ ReINHARD (wie Anm. 7) S. 26. Reinhard zieht den Begriff »networks« der Übersetzung "Netzwerke« vor.

"Sharon KeTTERING, Patrons, Brokers, and Clients in Seventeenth-Century France, New York, Oxford 1986. Reinhard wird von Kettering überhaupt nicht rezipiert. Ein methodisches Problem dieser Untersuchung besteht darin, daß Kettering vorwiegend Einzelfälle exemplifiziert, so daß die Klientelbildung vor allem für die Spitzen der Hierarchie - Richelieu, Mazarin -, weniger systematisch hingegen für die darunter liegende Ebene erschlossen wird. Mit verschiedenen Einzelaspekten von "Fidelity and Clientage in Seventeenth-Century France« setzen sich Beiträge von J. Russell MAJOR, Sharon KETTERING und Ellery SCHALK in den French Historical Studies XIV, 3 (1986) S. 391-446 auseinander. $\mathrm{Zu}$ unterschiedlichen Interpretationen in der »französischen« und der »angelsächsischen « Klientelforschung siehe den Artikel »Clientèles« von Arlette JOUANNA in: BÉLY (wie Anm. 3) S. 268-270. - Eine systematische Analyse von Patronage innerhalb einer gesellschaftlichen Gruppe Frankreichs, des Episkopats, liefert Joseph BERGIN, The Making of the French Episcopate 1589-1661, New Haven, London 1996. Innerhalb des Klientelsystems Mazarins spielte das Episkopat eine wesentliche Rolle, nicht allerdings im von uns zu untersuchenden Fall.

12 KeTtERING (wie Anm. 11) S. 181, 231. Auf die spezielle französische Situation geht auch Reinhard ein und analysiert, wie sich der Wechsel Mazarins vom Klienten innerhalb des römischen Patriziats zum Klienten Richelieus und dann zum Patron französischer und italienischer Klienten darstellt; REINHARD (wie Anm. 7) S. 33, 44, 69f.

${ }^{13}$ Verwandtschaft und andere Beziehungen spielten eine Rolle, wenn sie mit einer Klientelbeziehung einhergingen: Der französische Resident La Barde war zwar ein Neffe von Serviens Gegner Chavigny, verhielt sich aber politisch bewußt neutral, wie Servien in zwei Memoranden vom Mai 1646 Lionne berichtete; APW II B (wie Anm. 3), Bd. 3, bearb. v. Elke JARNUT und Rita BOHLEN mit einer Einleitung und einem Anhang von Franz BOSBACH, Münster 1999, nr. 262 und 292. Auch die Tatsache, daß La Barde sich in früheren Jahren einen Freund d'Avaux' genannt hatte, bewegte ihn nicht, zu dessen Gunsten in den Streit zwischen d'Avaux und Servien einzugreifen; vgl. dazu einen Brief La Bardes von 1639, zitiert in A[uguste] BOPPE, Correspondance inédite du Comte d'Avaux (Claude de Mesmes) avec son père, Paris 1887, S. 154 Anm. 2. - La Bardes Nachfolger Rorté war mit dem Trierer Kurfürsten Philipp von Sötern verwandt, ohne daß dies für ihn politisch bedeutsam wurde; Karlies ABMEIER, Der Trierer Kurfürst Philipp Christoph von 
Im frühneuzeitlichen Frankreich war - dies hat Sharon Kettering herausgestellt - die wichtigste Verbindung die zum respektive am Königshof ${ }^{15}$. Der Patron am Hof sicherte und verbesserte seine Position durch möglichst viele Klienten. Diese unterstützten ihn mit ihren - bescheideneren - Möglichkeiten und erwarteten von seiner Protektion ihren Aufstieg. Klientelbeziehungen waren allerdings völlig informell, anders als Lehensbeziehungen, die sie zum Teil ablösten, oder bürokratische Hierarchien. Wir können Klientelbeziehungen durch rhetorische Formulierungen ${ }^{16}$, Fremdzeugnisse ${ }^{17}$ oder anhand ihrer

Sötern und der Westfälische Friede (Schriftenreihe der Vereinigung zur Erforschung der Neueren Geschichte e.V., 15), Münster 1986, S. 141 und 144; APW (wie Anm. 3), Serie III C, Bd. 3 (Diarium Wartenberg), bearb. v. Joachim FOERSTER, 1988, S. 74 und 675. Serviens und Lionnes Klientelbeziehung zu Mazarin war eine lange Freundschaft vorausgegangen, die während der Fronde abkühlte. Dennoch blieben Lionne und Servien Klienten Mazarins; zu den Hintergründen siehe René KERVILER, Abel Servien, Le Mans 1877, S. 166f.; Anne-Marie ENAUX-MORET, Abel Servien, Paris 1968 (Thèse de l'École des chartes; zitiertes Exemplar: Archives Nationales Paris Ab XXVIII 121), S. $200 f$.

${ }^{14}$ Dies galt vor allem für Patrone, die nicht dem Hochadel entstammten und damit kaum auf traditionelle Anhänger ihrer Familie und nicht auf Klienten aus den Provinzen zurückgreifen konnten. D'Avaux warb häufig neue Klienten an, protegierte darunter aber auch Verwandte wie Henri d'Herbigny und Honoré Courtin, denen er während des Westfälischen Friedenskongresses diplomatische Aufgaben verschaffte. Servien war Klientelbeziehungen gegenüber mißtrauischer, nicht zuletzt wohl aufgrund schlechter Erfahrungen während der Regierung Richelieus. Er hielt seine Klientel zeitlebens klein und rekrutierte sie auffällig oft aus seinen Verwandten oder langjährigen Freunden: So förderte Servien seinen Neffen Lionne als politischen Vertrauten bereits während seiner Karriere in den 30er Jahren, und der französische Resident in Münster, La Court, der sein Amt Servien und Lionne verdankte, war ein angeheirateter Verwandter. Siehe dazu Anuschka TISCHER, Außenpolitik von Richelieu zu Mazarin. Französische Diplomatie und Diplomaten auf dem Westfälischen Friedenskongreß (Schriftenreihe der Vereinigung zur Erforschung der Neueren Geschichte e.V. 29), Münster 1999.

15 KeTTERING (wie Anm. 11) S. 141.

${ }^{16}$ Ein Kernbegriff der Patron-Klient-Rhetorik ist das »Interesse«, sei es in Form der Betonung gemeinsamen Interesses oder der Versicherung der Unterstützung von Interessen. Klienten boten oder versicherten sich einem Patron durch rhetorische Unterwerfungen, in der schärfsten Form in der Selbstbezeichnung als »Kreatur«, die auch Servien gegenüber Mazarin wählte. Darüber hinaus war die Rhetorik von übertriebener Höflichkeit und starker Emotionalität geprägt. Zur Rhetorik von Klientelbeziehungen siehe KETTERING (wie Anm. 11) S. 14f., 21f., 30; Antoni MACZAK, Patronage im Herzen des frühneuzeitlichen Europa, in: DERS., Klientelsysteme im Europa der Frühen Neuzeit (Schriften des Historischen Kollegs, Kolloquien, 9), München 1988, S. 83-89, hier S. 86. Servien gehörte zu den für die spätere Regierungszeit Mazarins typischen Klienten, die bereits eine versachlichte Rhetorik pflegten. Er argumentierte in einem Memorandum vom 18. Juni 1648, es sei nicht seine Aufgabe, angenehm zu schreiben, sondern nützlich zu informieren; Kopie: AE, CP Allemagne 117 fol. 263-268.

${ }^{17}$ Die erfolgreiche Klientelbeziehung, in der Patron und Klient sich zum gegenseitigen Vorteil unterstützten, war den Zeitgenossen in der Regel bekannt. So bezeichneten die kaiserlichen Botschafter in Münster in einem Brief an ihre Kollegen in Osnabrück Servien zutreffend als deß cardinals Mazzarini creatur; APW (wie Anm. 3), Serie II A (Kaiserliche Korrespondenz), Bd. 2, bearb. v. Withelm ENGELS, mit einem Nachtrag von Karsten 
Auswirkungen rekonstruieren, so daß jene Beziehungen, die weder dem Patron noch dem Klienten sichtbare Vorteile brachten oder aus denen nicht mindestens spürbare Unterstützung resultierte, der Historiographie häufig entgehen. Die Diplomatiegeschichte ist ein nahezu ideales Gebiet der Klientelforschung, denn bedingt durch die Distanz zum Hof schlugen sich Beziehungen, Patronage und die Konkurrenz verschiedener Klientelsysteme häufig schriftlich nieder, während der sonst übliche Weg, Kontakte zu knüpfen und Patronage zu praktizieren, das mündliche Gespräch war.

Der Einfluß von Klientelbeziehungen erreichte in Frankreich in der Zeit zwischen dem Tod Ludwigs XIII. 1643 und der Überwindung der Fronde um 1652 einen letzten Bedeutungshöhepunkt ${ }^{18}$. In dieser knappen Dekade, in die auch der Westfälische Friedenskongreß fällt, waren die verschiedenen Klientelsysteme bedeutend, nicht allein das auf den regierenden Minister hin geordnete. Der König war minderjährig; die Regentin Anna von Österreich stammte aus dem spanischen Königshaus, mit dem Frankreich sich im Krieg befand; ihr regierender Minister Mazarin war gebürtiger Italiener ${ }^{19}$. Ludwig XIII. hatte zudem die Regierung testamentarisch einem Regentschaftsrat übertragen, dessen Konstituierung Anna verhindern konnte ${ }^{20}$. So regierten mit Anna und Mazarin zwei Personen, die beide nicht in den französischen Führungsschichten und ihrer Anhängerschaft verwurzelt waren, sondern dort Gegner hatten, eine

RUPPERT, 1976, nr. 139 S. 269. Dennoch ist bei solchen Aussagen Vorsicht geboten, weil die Qualifizierung eines Gegners als Klient oder Kreatur eines anderen rasch bei der Hand war. Ein Beispiel für eine deutliche, jedoch nicht erfolgreiche Unterstützung eines Patrons durch seine Klienten war der Einsatz der Klienten Nicolas Foucquets zugunsten seiner Person nach seiner Verhaftung 1661, die zugleich das demonstrative Ende des Einflusses großer Klientelsysteme in Frankreich bedeutete; Julian DENT, The role of clientèles in the financial élite of France under Cardinal Mazarin, in: J. F. BOSHER ( $\mathrm{Hg}$.), French Government and Society 1500-1850. Essays in Memory of Alfred Cobban, London 1973, S. 41-69, hier S. 59.

${ }^{18}$ Ibid. S. $59 \mathrm{ff}$.

${ }^{19}$ Die Notwendigkeit des naturalisierten Franzosen Mazarin, sich gegen »nationale« Ressentiments zu verteidigen, spielte auch gegenüber den Botschaftern eine Rolle: In einem Schreiben an d'Avaux vom 12. Mai 1646 zeigte er sich zufrieden, daß die Spanier ihn zu harter Friedensbedingungen beschuldigten, klagten sie ihn doch damit an, d'estre fort bon François, comme effectivement je le suis; APW II B 3 (wie Anm. 13) nr. 265. D'Avaux formulierte dennoch in einem Brief an Mazarin vom 23. Oktober das süffisante Kompliment, er bewundere Mazarin als un seigneur estranger qui gouverne un grand royaume; APW II B (wie Anm. 3), Bd. 4, bearb. v. Clivia KELCH-RADE und Anuschka TISCHER, Münster 1999, nr. 216. Zu Mazarins Verteidigung gegenüber solchen Ressentiments siehe auch Lucien BÉLY, The Peace Treaties of Westphalia and the French Domestic Crisis, in: Heinz DuchHardT (Hg.), Der Westfälische Friede. Diplomatie - politische Zäsur - kulturelles Umfeld - Rezeptionsgeschichte, München 1998, S. 235-252, hier S. 240 f.

${ }^{20}$ Lloyd A. MOOTE, The Revolt of the Judges. The Parlement of Paris and the Fronde, Princeton (N. J.) 1971, S. 65; Orest RANUM, The Fronde, A French Revolution 1648-1652, New York, London 1993, S. 46ff. 
Gegnerschaft, die sich ab 1648 in den verschiedenen Ausläufern der Fronde entlud $^{21}$. Diese Gegner waren Franzosen mit allgemeinen Ressentiments gegen die Regierung zweier "Ausländer «; es waren Leute, die auf ein Ende der starken Regierung Richelieus nur gewartet hatten, um selbst politisch aktiv zu werden; und es waren die Mitglieder des verhinderten Regentschaftsrates, die größtenteils im Conseil d'en haut saßen.

Mazarin benötigte Unterstützung über die der Regentin hinaus und mußte vermeiden, sich weitere Kontrahenten zu schaffen. Aus einer Position der Stärke heraus konnte er nicht regieren. Nur so erklärt sich, wieso bei einem so bedeutenden außenpolitischen Ereignis wie dem Westfälischen Friedenskongre $\beta^{22}$ zwei französische Botschafter - Longueville und d'Avaux - Mazarin kritisch bis oppositionell gegenüberstanden und nur einer - sein Klient Abel Servien - ihm gegenüber loyal war. Diesen aber hatte Mazarin erst im letzten Moment in der Delegation plazieren können ${ }^{23}$, weil der vorgesehene Léon de Chavigny - auch dieser kein unbedingter Gewährsmann Mazarins - die Annahme der Mission verschleppte ${ }^{24}$.

Solange Mazarin keinen genügenden Rückhalt in der höfischen Administration besaß, unterstand die Politik nicht seiner unbedingten Kontrolle ${ }^{25}$. Der für

${ }^{21}$ Zur Ereignisgeschichte der Fronde siehe nach wie vor Ernst Heinrich KOSSMANN, La Fronde, Leiden 1954. Interpretatorische Neuansätze bietet RANUM (wie Anm. 20).

${ }^{22}$ Zum Westfälischen Frieden und Friedenskongreß allgemein siehe Konrad REPGEN, DreiBigjähriger Krieg und Westfälischer Frieden. Studien und Quellen, hg. v. Franz BosBACH und Christoph KAMPMANN, Paderborn u. a. 1998 (Rechts- und Staatswissenschaftliche Veröffentlichungen der Görres-Gesellschaft, Neue Folge, 81); DUCHHARDT (wie Anm. 19); Fritz DiCKMANN, Der Westfälische Frieden, hg. v. Konrad REPGEN, Münster ${ }^{7} 1998$; Heinz DUCHHARDr ( $\mathrm{Hg}$.), Bibliographie zum Westfälischen Frieden, bearb. v. Eva ORTLIEB und Matthias SCHNETTGER (Schriftenreihe der Vereinigung zur Erforschung der Neueren Geschichte e. V., 26), Münster 1996.

${ }^{23}$ Im September 1643 wurde Servien ernannt. Bereits zwei Monate später reisten er und d'Avaux zu Vorverhandlungen nach Den Haag; A[dolphe] CHÉRUEL ( $\mathrm{Hg}$.), Lettres du cardinal Mazarin pendant son ministère, 9 Bde., Paris 1872-1906, hier Bd. 1 S. 382. Vgl. auch IRSIGLER, Einleitung zu APW II B l (wie Anm. 3) S. LXIX.

${ }^{24}$ Chavigny und Mazarin hatten während der Regierung Richelieus ein freundschaftliches Verhältnis, das jedoch nach Richelieus Tod zunehmend in Konkurrenz umschlug. Bereits 1643 war Chavigny als Secrétaire d'État aux Affaires Étrangères durch Brienne ersetzt worden; vgl. auch DENT (wie Anm. 17) S. 48. Lionne und Servien fürchteten aber 1644 noch einen eventuellen Einfluß Chavignys auf Mazarin, wogegen Lionne intrigierte; APW II B 1 (wie Anm. 3), Beilage zu nr. 81, S. 159f. sowie nr. 109, S. 205.

${ }^{25}$ Paul Sonnino, Prelude to the Fronde. The French Delegation at the Peace of Westphalia, in: Heinz DuchHARDT (Hg.), Der Westfälische Friede. Diplomatie - politische Zäsur kulturelles Umfeld - Rezeptionsgeschichte, München 1998, S. 216-233, hier S. 219. Ein Memorandum Serviens vom 22. Juni 1648 hielt den Eindruck fest, Mazarins Anweisungen würden insgesamt unzureichend umgesetzt. Servien nannte keine Namen, verwies allerdings vor allem auf die sensiblen Bereiche der Finanzierung und der Kriegsführung und deutete an, die administrative Struktur des Hofes boykottiere Mazarins Regierung statt sie zu unterstützen; Kopie: AE, CP Allemagne 117 fol. 263-268. 
die Außenpolitik zuständige Secrétaire d'État, Henri-Auguste de Brienne, erwies sich als inkompetent ${ }^{26}$. Mazarin versuchte 1644, Briennes offiziellen Aufgabenbereich $\mathrm{zu}$ beschneiden, wogegen dieser sich aber erfolgreich zur Wehr setzte ${ }^{27}$. An eine Absetzung Briennes war gar nicht zu denken. Mazarin konnte ihn lediglich umgehen und tat dies mit Hilfe seines Sekretärs Hugues de Lionne ${ }^{28}$.

Für die politische Information und Kommunikation ergibt sich damit eine höchst verwirrende Situation: Regierungsbeschlüsse wurden offiziell im Conseil gefaßt und den Botschaftern durch Briefe Briennes mitgeteilt, ergänzt durch königliche Memoranden, die sowohl in Briennes wie Mazarins Kanzlei konzipiert wurden und die Brienne ausfertigen ließ und übersandte ${ }^{29}$. Darüber hinaus schrieb Mazarin Briefe in seinem eigenen Namen, die inoffiziell verbindlicher waren als jene Briennes. Auch in seinen eigenen Briefen nahm Mazarin aber Rücksicht auf den Conseil und während des Westfälischen Friedenskongresses auf zwei der drei Botschafter, nämlich Longueville und d'Avaux. Er hatte allerdings die einmalige Möglichkeit, seinen Klienten Servien, dem er anders als Longueville und d'Avaux kaum persönlich Briefe schrieb, völlig offen und ohne Risiko zu instruieren: Servien war der Onkel von Mazarins Sekretär Lionne. Beide führten eine Korrespondenz, die Mazarin - ohne offiziell in Erscheinung zu treten - nutzte, um seinen Klienten zu instruieren und um sich von ihm informieren zu lassen. Diese eigentlich private Korrespondenz war hochpolitisch, denn dort findet man häufiger als an anderer Stelle Mazarins ungeschminkte Ansichten ${ }^{30}$.

${ }^{26}$ Brienne war für eine administrative Karriere ausgebildet worden und hätte sich wegen früher Auslandsaufenthalte für das auswärtige Ressort durchaus eignen können. Tatsächlich sind einige seiner Stellungnahmen sehr scharfsinnig, doch argumentierte er gewöhnlich langatmig und konfus. Zu Briennes Person und Werdegang siehe Louis LÉVÊQUE, Le comte de Brienne, in: Revue Historique 35 (1910) S. 40-264. Daß Briennes Anweisungen oft auch für die Diplomaten unverständlich waren, belegen mehrere Schreiben Serviens von 1645 und 1646; APW II B 2 (wie Anm. 5) nr. 14, S. 64; ibid. nr. 106, S. 359; APW II B 3 (wie Anm. 13) nr. 40.

${ }^{27}$ Darüber berichtete Lionne Servien am 30. April 1644; APW II B 1 (wie Anm. 3), Beilage zu nr. 81, S. 160.

${ }^{28}$ Paulette ENJALRAN, Marie-Thèrèse DENIS-COMBET, L'Ancien Régime, in: Jean BAILlou (Hg.), Les Affaires Étrangères et le Corps Diplomatique Français, Bd. I, Paris 1984, S. 13-272, hier S. $81 \mathrm{ff}$; Derek CROXTON, Peacemaking in Early Modern Europe: Cardinal Mazarin and the Congress of Westphalia, 1643-1648, Selinsgrove, London 1999, S. 36f.; MOOTE (wie Anm. 20) S. 70. Durchgreifende Aktionen gegen Brienne wurden auch dadurch erschwert, daß er das besondere Vertrauen Königin Annas genoß.

${ }^{29}$ Zum Geschäftsgang siehe IRSIGLER, Einleitung zu APW II B I (wie Anm. 3) S. LXXIXff.; Anne-Marie ENAUX, Les plénipotentiaires français en Westphalie, in: 1648 - la paix de Westphalie vers l'Europe moderne (Katalog zur Ausstellung im Hôtel de la Monnaie, Paris, 16. September-31. Oktober 1998), Paris 1998, S. 125-134, hier S. 127.

${ }^{30}$ Dies zeigte sich deutlich in der Zeit der Jahreswende 1647/48, als sich ein Scheitern der Verhandlungen mit Spanien abzeichnete, während die innerfranzösische Opposition ver- 
Persönliche Beziehungen übten also Einfluß auf die französische Diplomatie des Westfälischen Friedenskongresses aus, was auch vermeintliche Ressortkompetenz überlagern konnte. Betrachten wir im folgenden jene drei Männer, die diese Diplomatie vertraten: die Botschafter Longueville, d'Avaux und Servien $^{31}$.

Henri von Orléans, Herzog von Longueville (1595-1663), gebürtig aus einer illegitimen Linie des französischen Königshauses, wurde, obwohl er nie zuvor diplomatisch tätig gewesen war, aufgrund seines gesellschaftlichen Ranges französischer Prinzipalbotschafter. Ein Herzog von Longueville war in einer Klientelbeziehung nicht anders denn als Patron und einzig dem König untergeordnet denkbar. Er besa $\beta$ durch seine Herkunft und als Gouverneur der Normandie ein Klientelsystem, dessen innenpolitische Bedeutung von nur wenigen anderen erreicht wurde ${ }^{32}$. Wäre der Herzog allerdings eine Generation früher geboren, seine Position wäre weitaus besser gewesen: Es waren hohe Schwertadelige wie Longueville, welche nach wie vor die Provinzgouvernements innehatten. Diese Gouvernements waren bis ins 17. Jahrhundert hinein zentrale Machtpositionen, wenn sie auch den Höhepunkt ihrer Bedeutung mit den Religionskriegen überschritten hatten. Doch alle Kontakte zwischen der Provinz und dem Hof - von der Vertretung von Interessen bis zur Vermittlung von Personen - liefen zunächst weiterhin über den Gouverneur. Der Gouverneur stand somit a priori an der Spitze eines potentiellen Klientelsystems beträchtlichen Ausmaßes. Genau diese Position der Gouverneure aber untergrub Richelieu, indem er eigene Strukturen schuf, welche den Hof mit den Provinzen in direkten Kontakt brachten ${ }^{33}$. In der französischen Gesandtschaft auf

stärkt auf den Friedensschluß drängte. Mazarin wies Servien durch Lionne am 31. Dezember 1647 an, sich in diesen Verhandlungen weniger kompromißbereit zu zeigen, als es die Instruktionen zuließen; Ausfertigung: AE, CP Allemagne 103 fol. 511-511'. Am 11 . Januar 1648 teilte Lionne Servien mit, daß Mazarin jeden Kompromiß über Herzog Karl von Lothringen, an dem der Verhandlungserfolg zeitweilig zu hängen schien, ablehne, eine offene Stellungnahme gegenwärtig aber scheue; Ausfertigung: AE, CP Allemagne 118 fol. 63. Vorwürfe, Servien besitze geheime Instruktionen, gab es schon während des Kongresses, so vom Mediator Alvise Contarini; dazu APW II B 4 (wie Anm. 19) nr. 152. Mazarin leugnete aber noch 1651 in einem Brief an Brienne, Servien einen Informationsvorsprung verschafft zu haben; Lettres du cardinal Mazarin (wie Anm. 23) Bd. 4, S. 94f.

${ }^{31}$ Für ausführlichere biographische Informationen über die drei Botschafter, insbesondere zu ihrem sozialen und politischen Hintergrund und ihren diplomatischen Differenzen während des Westfälischen Friedenskongresses, siehe TISCHER (wie Anm. 14); vgl. auch ENAUX (wie Anm. 29). Eine veröffentlichte wissenschaftliche Monographie oder längere Abhandlung liegt zu keinem der drei vor.

${ }^{32}$ Erst während der Fronde gelang es Mazarin, dieses Klientelsystem zu überwinden. Siehe dazu Madeleine FolsIL, Parentèles et fidélités autour du duc de Longueville, in: Yves DURAND (Hg.), Hommage à Roland Mousnier: Clientèles et fidélités en Europe à l'Epoque moderne, Paris 1981, S. 153-168.

${ }^{33}$ Zur sich wandelnden Rolle sowie zur sozialen Herkunft der Gouverneure siehe grundlegend Robert R. HARDING, Anatomy of a Power Elite. The Provincial Governors of Early 
dem Westfälischen Friedenskongreß befanden sich zwei Personen, die davon bereits profitiert hatten: Botschafter Servien aus der Dauphiné und Resident La Court aus Longuevilles eigener Provinz Normandie war in einer administrativen Klientelbeziehung - Servien zunächst über Richelieu, dann über Mazarin, La Court wiederum über Servien und Lionne - ein Aufstieg gelungen, den sie früher nicht oder eben nur über den Gouverneur geschafft hätten ${ }^{34}$.

Dennoch war das Klientelsystem Longuevilles kein geringer Faktor: Einerseits besaß Mazarin noch nicht Richelieus Verbindungen in die Provinzen und vernachlässigte diese zunächst sogar, während er seine Beziehungen in Paris auf- und ausbaute ${ }^{35}$. Andererseits war der Herzog ein latenter Oppositioneller, der nach Regierungsbeteiligung strebte, diese aber auch nach dem Tod Ludwigs XIII. und Richelieus nicht erlangte. So gehörte auch er zu den Gegnern der Regentschaftsregierung, und seine Entsendung nach Münster bedeutete zugleich seine Entfernung aus dem politischen Zentrum.

Longueville wollte seine innenpolitische Stellung weiter ausbauen. Von Münster aus scheiterten allerdings 1646 zwei seiner Versuche, bedeutende Ämter zu übernehmen ${ }^{36}$. Er prognostizierte Aufstände gegen Mazarin und pflegte offen persönliche Kontakte zum Kreis um die berüchtigte Herzogin von Chevreuse, nachdem diese 1645 erneut aus Frankreich geflüchtet war $^{37}$.

Modern France, New Haven, London 1978. Vgl. daneben Roger METTAM, Power and Faction in Louis' XIV France, New York 1988, S. 47-54, 66; KETTERING (wie Anm. 11) S. 141-144.

${ }^{34}$ Wie sehr dies noch das Denken prägte, zeigt sich daran, daß La Court, der von Longueville in keiner Weise protegiert worden war, nach seiner Ernennung zum Residenten dennoch versicherte, die Mitglieder seiner Familie seien immer treue Diener der Gouverneure der Normandie gewesen; La Court an Servien, Paris 1646 Juni 29; APW II B 4 (wie Anm. 19) nr. 39.

${ }^{35}$ KeTTERING (wie Anm. 11) S. 142f., 161.

${ }^{36}$ Zunächst meldete er nach dem Tod des Herzogs von Brézé in der französisch-spanischen Seeschlacht vor Orbetello am 14. Juni 1646 seinen Anspruch auf das freigewordene Gouvernement von Brouage und die Surintendance de la navigation et du commerce an, wenige Monate später auf das Amt eines Colonel général des Suisses et Grisons. Zu den Auseinandersetzungen um die Brézé-Nachfolge siehe TISCHER, Einleitung zu APW II B 4 (wie Anm. 19) mit den dort zitierten Schreiben. Daß Longuevilles vergebliche Versuche, seine innenpolitische Position auf diesem Wege auszubauen, von einem breiten Personenkreis wahrgenommen wurden, belegt ein Schreiben von Serviens Privatkorrespondent Jacques Gaudin vom 29. Juni 1646 aus Paris; Ausfertigung: AE, Série Mémoires et Documents (MD) France 855 fol. 145-146.

${ }^{37}$ Die Flucht der Herzogin, die bereits zu Lebzeiten Ludwigs XIII. im Exil gelebt hatte, erfolgte wenige Monate vor Longuevilles Abreise vom Hof und wurde d'Avaux und Servien durch ein Schreiben Briennes vom 22. April 1645 offiziell mitgeteilt; APW II B 2 (wie Anm. 5) nr. 85, S. 289. Während Longueville in Münster weilte, versuchte die Herzogin von Chevreuse, ihn in ihren oppositionellen Kreis zu ziehen. Sie benutzte dazu vor allem ihren Vertrauten Henri de Saint-Ibard, der auch zwischen ihr und der spanischen Seite Kontakt hielt und zu persönlichen Gesprächen mit Longueville nach Münster reiste. Darüber bzw. über Longuevilles Prognosen, es werde in zwei oder drei Jahren zu Auf- 
Vor allem wollte Longueville den Kongreß möglichst schnell wieder verlas$\operatorname{sen}^{38}$, was man ihm im Februar 1648 schließlich nicht mehr verweigern konn$\mathrm{te}^{39}$.

Es war für Mazarin ein großes Glück, keine Selbstverständlichkeit, daß Longueville, der später während der Fronde ein Bündnis mit Spanien einging, sich auf dem Kongreß weder von den Kriegsgegnern vereinnahmen ließ noch eigene außenpolitische Zielvorstellungen einbrachte. Longueville war 1643 ernannt worden, hatte aber erst im Sommer 1645 nach Münster reisen müssen, auch weil zwischen den bereits anwesenden Botschaftern d'Avaux und Servien ein Streit eskalierte, der sich nur noch durch seine Anwesenheit bewältigen lie $3^{40}$. Dieser Streit, von dem noch die Rede sein wird, schwelte während des gesamten Kongresses. Dadurch hätte Longueville eine einflußreiche Rolle zuwachsen können. Doch er war keine eigenständige diplomatische Persön-

ständen gegen die Regentin kommen, berichtete Servien Lionne am 20. April und am 17. Juli 1646; APW II B 3 (wie Anm. 13) nr. 230 und APW II B 4 (wie Anm. 19) nr. 75. Vgl. auch Louis BATIFFOL, La duchesse de Chevreuse, Paris 1913, S. 230f. Mazarin warnte Longueville vor Saint-Ibard, doch der Herzog verteidigte seine freundschaftliche Beziehung zu ihm; Mazarin an Longueville, [Montdidier] 1646 Mai 29; APW II B 3 nr. 289; Longueville an Mazarin, 1646 Juni 13; APW II B 4 nr. 7. Vgl. auch BÉLY (wie Anm. 19) S. 247, Anm. 34.

${ }^{38}$ Im Sommer 1647 versuchte Longueville mehrmals erfolglos, seine Abreise durchzusetzen. Siehe dazu: Mazarin an Longueville, 1647 Juni 8; Lettres du cardinal Mazarin (wie Anm. 23) Bd. 2, S. 437f.; Servien an Lionne, 1647 August 13; AE, CP Allemagne 101 fol. 259-262; Longueville an Mazarin, Münster 1647 August 19; Ausfertigung: AE, CP Allemagne 85 fol. 76-76'; Mazarin an Longueville, 1647 August 30; Kopie: AE, CP Allemagne 101 fol. 360-360'; Longueville an Mazarin, Münster 1647 September 9; Ausfertigung: AE, CP Allemagne 85 fol. 176-177.

${ }^{39}$ Mazarin erteilte Longueville am 3. Januar 1648 die offizielle Erlaubnis abzureisen (Lettres du cardinal Mazarin [wie Anm. 23] Bd. 3 S. 1), versuchte aber noch mit einem Brief vom 31. Januar, ihn auf dem Kongreß zu halten (Kopie: AE, CP Allemagne 118 fol. 274-276). Daß es Longuevilles eigene Entscheidung war, den Kongreß zu verlassen, bezeugt auch ein Schreiben seines Sekretärs und Dolmetschers Stenglin an seinen Korrespondenten Stotz vom 1. Februar 1648; Kopie: Burgerbibliothek Bern, Manuskripte, Serie Historica Helvetica XV 37, S. 156-157.

${ }^{40}$ D'Avaux und Servien tauschten im Rahmen ihres Streits Briefe aus, die teilweise als Flugschriften verbreitet und bereits 1650 ohne Orts- und Verlagsangabe erstmals publiziert wurden: Lettres De Messieurs d'Avavx Et Servien, Ambassadevrs Pour le Roy de France en Allemagne, concernantes leurs differents \& leurs reponses de part \& d'autre en l'Année 1644 (zitiertes Exemplar: BNF Lg 6.161). Diese Kompilation, die trotz des Titels Briefe über das Jahr 1644 hinaus enthält, wurde letztmalig geschlossen abgedruckt in den Négociations secrètes touchant la paix de Munster et d'Osnabrug, Bd. 1, Den Haag (Jean Neaulme) 1725. Vgl. auch E[mile] CHARvÉRIAT, D'Avaux \& Servien, in: Revue du Lyonnais, Série $5 \mathrm{~N}^{\circ} 4$ (1888) S. 252-258. Zahlreiche Quellen zu diesem Streit finden sich in APW II B 1 (wie Anm. 3) und 2 (wie Anm. 5). Eine Revision der Auseinandersetzung unter Berücksichtigung neuer Quellenfunde erfolgt in TISCHER (wie Anm. 14). D'Avaux hatte sich 1645 bereits entschlossen gezeigt, den Kongreß zu verlassen, und revidierte den Entschluß erst, als das Kommen Longuevilles gesichert war, wie er dem Herzog am 6. Juni 1645 aus Münster schrieb; Kopie: BNF, Coll. Baluze 172 fol. 256. 
lichkeit: In Zweifelsfällen holte er lieber nochmals höfische Instruktionen ein. Und als er 1647 einige Monate ohne seine Kollegen in Münster verhandelte, weil Servien zu einer Mission in Den Haag, d'Avaux in Osnabrück weilte, hatte er die Verhandlungen bald festgefahren. Er bat darum, daß d'Avaux aus Osnabrück zurückkehren möge, ein Eingeständnis, daß er sich alleine die Verhandlungsführung nicht länger zutraute ${ }^{41}$.

Die beiden französischen Botschafter, die tatsächlich den Wunsch hatten, Außenpolitik und Diplomatie auf dem Westfälischen Friedenskongreß zu beeinflussen, und dabei ihre Beziehungen nachdrücklich nutzten, waren d'Avaux und Servien.

Claude de Mesmes, Graf d'Avaux (1595-1650) hatte bei Kongreßbeginn etwas eher Seltenes hinter sich: eine 17jährige Karriere als französischer Botschafter in zahlreichen europäischen Staaten. Er strebte durchaus nach einer höfischen Karriere ${ }^{42}$, war aber kein Klient Richelieus ${ }^{43}$. Seine Tätigkeit wurde zwar 1638 mit der Erhebung seiner Herrschaft Avaux zur Grafschaft honoriert $^{44}$, einen Sitz im Conseil und das Amt eines Surintendant des finances konnte er aber erst nach Richelieus Tod erlangen ${ }^{45}$. Grundsätzlich hatte d'Avaux gute Voraussetzungen: Er stammte aus einer renommierten amtsadeligen Pariser Familie; sein Vater verkehrte am Hof; zwei seiner Brüder saßen auf hohen Positionen im Pariser Parlament ${ }^{46}$; er war vermögend ${ }^{47}$; und: er

${ }^{41}$ Longueville an Servien, 1647 April 12; Ausfertigung: AE, CP Allemagne 99 fol, $331-$ 333; ders. an d'Avaux, 1647 April 13; Ausfertigung: AE, CP Allemagne 88 fol. 97-97'. Kritik an Longuevilles Vorgehensweise hatten zuvor Brienne in einem an den Herzog gerichteten Brief vom 8. Februar (Kopie: AE, CP Allemagne 98 fol. 281-283') und Mazarin in einem vom 22. März ( $\mathrm{AE}, \mathrm{CP}$ Allemagne 99 fol. 210-214') geübt.

${ }^{42}$ Sein Mittelsmann am Hof war Père Joseph, Richelieus "graue Eminenzu. Siehe dazu einen Brief Père Josephs an d'Avaux von 1635 in François PULASKI, Ladislas TOMKIEWICZ (Hg.), La Mission de Claude de Mesmes Comte d'Avaux, Ambassadeur Extraordinaire en Pologne 1634-1636 (Documents relatifs aux Rapports Diplomatiques entre la France et la Pologne, 1), Paris 1937, nr. 33 S. 104 ff.

${ }^{43}$ Zur Klientelpolitik Richelieus als Minister siehe neben RANUM (wie Anm. 9) auch Lloyd A. Moote, Richelieu as Chief Minister, in: Joseph BERGIN, Laurence BROCKLISS (Hg.), Richelieu and his Age, Oxford 1992, S. 13-43; Klaus MALETTKE, The Court of King Louis XIII, in: Ronald G. ASCH, Adolf M. BIRKE (Hg.), Princes, Patronage and the Nobility, Oxford 1991, S. 415-439, hier S. 432-439.

44 PULASKI, TOMKIEWICZ (wie Anm. 42) S. 105.

${ }^{45}$ SONNINO (wie Anm. 25) S. 218. Es war nach Richelieus Tod bereits das zweite Regierungsamt, um das d'Avaux sich bemühte: Bei der Bewerbung um das Amt als Secrétaire d'État à la guerre hatte sich Michel Le Tellier gegen ihn durchgesetzt; Louis ANDRÉ, Michel Le Tellier et l'organisation de l'armée monarchique, Paris 1906, S. 96.

${ }^{46}$ Zum familiären Hintergrund d'Avaux' siehe François BLANCHARD, Les Presidens av Mortier dv Parlement de Paris, Paris 1647 [zitiertes Exemplar: BNF Lf 25.30; das im Titel genannte Erscheinungsjahr kann nicht stimmen, da die Angaben in dem Werk bis in das Jahr 1649 reichen], S. 387-397 (1. Teil).

${ }^{47} \mathrm{Vgl}$. dazu auch Anm. 51 . 
pflegte freundschaftliche Beziehungen bis in den Conseil hinein ${ }^{48}$. Angesichts seiner guten Voraussetzungen hatte er offensichtlich keine Neigung, sich um einen Patron zu bemühen, wenn auch einflußreiche Persönlichkeiten sich hin und wieder seiner Interessen annahmen ${ }^{49}$. D'Avaux blieb eine eigenständige politische Persönlichkeit. Und als solche hatte er während der Regierung Richelieus keine Chance, eine höfische Karriere zu machen. Daß d'Avaux unter Mazarin wiederum Botschafter wurde, war zunächst - anders als bei Longueville - keine politische Exilierung. D'Avaux war schon 1635 von Richelieu als Unterhändler für einen künftigen Friedenskongreß ausersehen worden ${ }^{50} . \mathrm{Er}$ residierte mehrere Jahre im Reich und handelte die Kongreß-Präliminarien aus. Es galt als selbstverständlich, daß er Frankreich auf dem Kongreß vertreten würde.

D'Avaux besaß einen starken Rückhalt in Paris. Aber seine Beziehungen und das Klientelsystem, das er aufbaute, waren noch mehr von seiner langen Zeit als Botschafter geprägt. Er hatte Freunde an fremden Höfen und unter ausländischen Diplomaten. Darunter befanden sich in der schwedischen Kongreßgesandtschaft in Osnabrück der Botschafter Salvius, der bei d'Avaux hoch verschuldet war $^{51}$, und der Sekretär und spätere Resident Mylonius Biörenklou ${ }^{52}$. Diese Beziehungen machten sich während des Streits zwischen d'Avaux und Servien 1645 bemerkbar, als die schwedischen Botschafter ge-

${ }^{48}$ Zu d'Avaux' Beziehungen während der 30er Jahre siehe BOPPE (wie Anm. 13) S. 88, 206. In Münster führte d'Avaux eine, wenn auch nicht intensive, Einzelkorrespondenz mit dem Herzog von Orléans und Mitgliedern der Familie Enghien-Condé. Briefe aus dieser Korrespondenz finden sich AE, CP Allemagne 66 fol. 213-213' und 437; AE, CP Allemagne 67 fol. 159-160 und 162-163'. Lionne, der d'Avaux' besondere Beziehungen zu den Princes du sang mit Mißtrauen beobachtete, berichtete Servien zudem in einem Brief vom 31. Dezember 1647, daß sich eine Nichte d'Avaux', Jeanne-Marie-Angélique de Fouquesolles, im Gefolge der Tochter des Herzogs von Orléans befinde; Ausfertigung: AE, CP Allemagne 101 fol. 367-369.

${ }^{49} \mathrm{Zu}$ den Bemühungen des 1638 verstorbenen Père Joseph vgl. Anm. 42. Nach d'Avaux' Rückkehr aus Münster 1648 setzte sich Condé verstärkt für ihn ein, was d'Avaux zusätzliches Mißtrauen von Königin Anna eintrug; Mémoires de Mathieu de Molé, bearb. v. Aimé Champollion-FigeaC, Bd. IV, Paris 1857, S. 406.

${ }^{50}$ Anja Victorine HaRTMANN, Von Regensburg nach Hamburg. Die diplomatischen Beziehungen zwischen dem französischen König und dem Kaiser vom Regensburger Vertrag (13. Oktober 1630) bis zum Hamburger Präliminarfrieden (25. Dezember 1641) (Schriftenreihe der Vereinigung zur Erforschung der Neueren Geschichte e. V., 27), Münster 1998, S. 199.

51 Salvius an Königin Christina, 1648 März 13/23; APW (wie Anm. 3), Serie II C (Schwedische Korrespondenz), Bd. 4, bearb. v. Wilhelm KoHL unter Mitarbeit von Paul NACHTSHEIM, 1994, nr. 181 S. 335f.; d'Avaux an Salvius, 1650 Mai 26; Konzept: BNF, Coll. Baluze 172 fol. 301-301'. Die Schulden beliefen sich auf 50000 Reichstaler.

52 D'Avaux an Longueville, [Osnabrück] 1647 Januar 23; Duplikat: AE, CP Allemagne 98 fol. 171-174. 
gen eine mögliche Abreise d'Avaux' intervenierten und sich so hinter ihn stell$\operatorname{ten}^{53}$.

Vor allem stand d'Avaux als Patron an der Spitze eines Klientelsystems, das - bedingt durch seine Tätigkeit - teilweise deckungsgleich war mit Frankreichs diplomatischem Corps. So richteten 1643/44 die französischen Residenten im Reich ihre Berichte zunächst nur an d'Avaux, nicht an seinen Kollegen Servien $^{54}$. Die beiden französischen Kongreß-Residenten, Saint-Romain in Münster und Rorté in Osnabrück, waren unbedingte Klienten d'Avaux', die schon sechs Jahre mit ihm zusammengearbeitet hatten und auf ihn ausgerichtet waren $^{55}$. Diese Personenkonstellation ließ Servien als gleichberechtigten Botschafter neben d'Avaux zunächst nicht zu und produzierte ein Übergewicht an offiziellen Berichten zugunsten d'Avaux ${ }^{56}$.

Für Servien war es schon deshalb schwierig, d'Avaux' diplomatische Klientel anzugreifen, weil Angriffe auf außenpolitische Funktionsträger zugleich Brienne trafen, den langjährige gute Beziehungen mit d'Avaux verbanden und unter dessen Mitarbeitern d'Avaux Anhänger besa $\beta^{57}$. Mit Hilfe einer Intrige Lionnes konnte Servien Rorté im Juli 1645 entfernen lassen ${ }^{58}$, Saint-Romain

${ }^{53}$ Lionne an Servien, Paris 1645 Mai 20; APW II B 2 (wie Anm. 5) nr. 104 S. 351. Mazarin betonte darüber hinaus, daß das bestehende Vertrauensverhältnis zwischen d'Avaux und Salvius positive Impulse auf die Verhandlungen erwarten lasse; APW Il B 3 (wie Anm. 13) nr. 127

${ }^{54}$ IrSigleR, Einleitung zu APW Il B 1 (wie Anm. 3) S. LXX. Vgl. auch ENAUX (wie Anm. 29) S. 126.

${ }^{55}$ Diese Phase der Zusammenarbeit ist dokumentiert in dem von BOPPE (wie Anm. 13) edierten Briefwechsel d'Avaux' mit seinem Vater. Saint-Romain und Rorté waren beide zu Residenten auf dem Kongreß ernannt worden, bevor Servien Botschafter wurde, so daß sie die ersten Richtlinien für ihre Tätigkeit ebenfalls von d'Avaux erhielten; siehe dazu Schreiben d'Avaux' für Saint-Romain vom 24. September 1642 (Konzept: BNF Coll. Baluze 172 fol. 74-76, Ergänzung fol. 73') und für Rorté vom 20. Juni 1643 (Ausfertigung: BNF Fonds Français 15935 fol. 454).

${ }^{56}$ Saint-Romain und Rorté korrespondierten auch miteinander und an Servien vorbei über die Verhandlungen; siehe dazu einen Brief Saint-Romains an Rorté vom 30. Dezember 1644; Ausfertigung: Institut de France, Paris, Coll. Godefroy 273 fol. 310-311'. Daß die Parteiungen innerhalb der Gesandtschaft in Paris bekannt waren, belegt ein Brief Briennes an d'Avaux vom 9. Juli 1644; APW II B 1 (wie Anm. 3) nr. 162 S. 337.

${ }^{57}$ Nach massiven Vorwürfen Serviens gegen Klienten d'Avaux' beklagte Brienne sich bei d'Avaux, er wünsche, que Monsieur Servien eust meilleure opinion de ceux qui servent le Roy soubz moy; ibid. nr. 193 S. 427. Zum freundschaftlichen Verhältnis Briennes zu d'Avaux siehe Briennes Schreiben vom 18. Juni 1644 (ibid. nr. 143 S. 274) und vom 4. Februar 1645 (APW II B 2 [wie Anm. 5] nr. 32 S. 115). Zur Anhängerschaft d'Avaux' unter den Mitarbeitern des Staatssekretärs siehe ein Schreiben Lionnes vom 2. Juli 1646: $J$ 'ay descouvert que monsieur d'Avaux a gagné tous les commis de monsieur de Brienne, et qu'ilz font grand fondement sur son retour comme s'il devoit les enrichir tous; APW II B 4 (wie Anm. 19) nr. 47.

${ }^{58}$ Siehe dazu die Schreiben Serviens an Lionne vom [15.] Januar 1645 (APW Il B 2 [wie Anm. 5] nr. 14 S. 60), Mazarins an d'Avaux und Servien vom 28. Januar (ibid. nr. 24 S. 88) sowie Serviens an Lionne vom 14. Juli (ibid. nr. 165 S. 513). 
aber reiste erst mit d'Avaux im April $1648 \mathrm{ab}^{59}$. Servien und Lionne verhinderten, daß d'Avaux Saint-Romain einen besseren Posten verschaffte oder ihn als seinen Nachfolger im Reich vorsah, um sich langfristigen außenpolitischen Einfluß zu sichern ${ }^{60}$. Aber Saint-Romain blieb bis 1648 ein parteilicher Informant, der mit Mazarin und besonders intensiv mit Chavigny über die Verhandlungen korrespondierte ${ }^{61}$, während Servien vergeblich bestritt, daß ein Resident in Münster überhaupt erforderlich $\mathrm{se}^{62}$. Ohne diplomatischen Nutzen war Saint-Romain nicht, denn er übernahm mehrmals kleinere Missionen, die d'Avaux wiederum besser kontrollieren konnte und über die er besser informiert wurde ${ }^{63}$.

D'Avaux' Voraussetzungen hätten ihm die Führung in den Verhandlungen sichern können, denn er besaß neben seinen Verbindungen langjährige Erfahrungen in der Materie, Sprachkenntnis ${ }^{64}$ und andere diplomatische Qualifikationen, die Servien nicht hatte ${ }^{65}$. Dennoch zog dieser die Verhandlungen an sich, und d'Avaux wurde vorzeitig abberufen.

${ }^{59}$ Die Abberufung Saint-Romains gemeinsam mit d'Avaux war zwar naheliegend, ging jedoch auch auf Saint-Romains eigenen Wunsch zurück, da er, wie er in einem Brief an den Hof vom 2. März 1648 versicherte, das Amt nur unter d'Avaux ausüben wollte; Ausfertigung: AE, CP Allemagne 107 fol. 284-285'.

${ }^{60}$ Memorandum Serviens für Lionne, Münster 1646 April 14; APW II B 3 (wie Anm. 13) nr. 222; Lionne an Servien, Paris 1646 Mai 5; ibid. nr. 256. D'Avaux bemühte sich zweimal, nach der Abberufung Rortés 1645 und der La Bardes 1646, Saint-Romain zum Residenten in Osnabrück ernennen zu lassen. Für Saint-Romain begann erst 1666 eine Botschafter-Karriere. Zu seiner Person siehe Antoine VACHEZ, Melchior Arod (M. de Saint Romain), nach dem Tod des Autors hg. v. Roger VILlE, Lyon 1913 (zitiertes Exemplar: BNF $8^{\circ}$ Ln 27.58582).

${ }^{61}$ Zahlreiche Briefe Saint-Romains aus dieser Korrespondenz finden sich in den Archivbänden $\mathrm{AE}, \mathrm{CP}$ Allemagne 61, 62, 67, 87-90, 107. Insbesondere in den letzten Monaten vor d'Avaux' und Saint-Romains Abberufung berichtete der Resident in einem zunehmend aggressiveren Ton gegen Servien. Vgl. dazu seine Briefe vom 31. Dezember 1647 und vom 15. Januar 1648; Ausfertigung: AE, CP Allemagne 90 fol. 316-320' und AE, CP Allemagne 107 fol. 52-53.

62 Servien an Lionne, [Münster] 1646 November 20; APW II B 4 (wie Anm. 19) nr. 265.

${ }^{63} \mathrm{Vgl}$. auch Anm. 83. Im Oktober 1646 kehrte Saint-Romain von Osnabrück nach Münster zurück und erstellte ein Memorandum über neue Entwicklungen in den kaiserlichschwedischen Verhandlungen, an denen er beteiligt gewesen war. Dieses Memorandum wurde in einer gemeinsamen Depesche der Botschafter am 8. Oktober, als Beilage $3 \mathrm{zu}$ APW II B 4 (wie Anm. 19), an den Hof gesandt. Dennoch beklagte Servien sich bei Lionne, von dem Inhalt des Memorandums keine Kenntnis zu haben; ibid. nr. 192.

${ }^{64} \mathrm{Zu}$ den unterschiedlichen Sprachkenntnissen der Botschafter siehe auch den Beitrag von Guido Braun in diesem Band.

${ }^{65}$ Während einer Debatte im Pariser Parlament am 17. Dezember 1648 bezweifelte der Parlamentsrat Claude Aubry, daß Servien alleine mit Spanien erfolgreich den Frieden verhandeln könne, nicht nur, weil es dazu mehrerer Unterhändler bedürfe, sondern auch, weil des personnes de probité, suffisance et naissance entsandt werden müßten; Jean LE BOINDRE, Débats du Parlement de Paris pendant la minorité de Louis XIV, hg. v. Robert Descimon, Orest Ranum und Patricia M. Ranum, Paris 1997, S. 285. 
Abel Servien (1593-1659) stammte wie d'Avaux aus einer renommierten amtsadeligen Familie. Doch er kam aus der Dauphiné, war ohne nennenswerte Kontakte zum Hof ${ }^{66}$, ohne größeres Vermögen ${ }^{67}$ und führte erst durch seine Gattin seit 1641 den konstruierten Titel eines Grafen von La Roche des Aubiers $^{68}$. Er verdankte seinen Aufstieg der Tatsache, sich bereits um 1615 Richelieu angeschlossen zu haben. Als dieser 1624 Minister Ludwigs XIII. wurde, begann für Servien eine rasche, teilweise auch diplomatische Karriere ${ }^{69}$. Als Klient Richelieus brachte er es in nicht einmal zehn Jahren bis zum Secrétaire d'État à la guerre. Nach einem Zusammenspiel von eigenem Fehlverhalten und fremden Intrigen mußte Servien jedoch 1636 sein Amt aufgeben und den Hof verlassen ${ }^{70}$. Ohne Richelieus Unterstützung hatte Servien keinerlei Rückhalt.

Servien hatte jedoch mit Mazarin früh, Ende der 20er Jahre, Freundschaft geschlossen ${ }^{71}$. Nach Richelieus Tod bot er sich ihm sofort und unbedingt als Klient an und unterstellte sich in seinen Briefen als créature völlig Mazarins Willen $^{72}$. Für Mazarin war Servien allerdings kein besonders attraktiver Klient,

${ }^{66}$ Servien resümierte selbst seine sozialen Nachteile in einem Brief an Brienne vom 13. August 1644; APW II B 1 (wie Anm. 3) nr. 205 S. 448.

${ }^{67}$ Es war ein Streitpunkt zwischen d'Avaux und Servien, daß Servien nicht mit dem von seinem Kollegen betriebenen finanziellen Aufwand mithalten konnte; Négociations secrètes touchant la paix de Munster et d'Osnabrug (wie Anm. 40) Bd. 1, S. 87f. Mazarin ließ Servien mehrfach Sonderzahlungen zukommen, darunter eine finanzielle Entschädigung für die Einnahmen, die ihm durch seine Amtsenthebung seit 1638 entgangen waren; Kopie (datiert auf 1645) eines Conseil-Beschlusses von 1643: AE, MD France 852 fol. 295. Servien konnte aber erst nach seiner Rückkehr aus Münster, insbesondere als $\mathrm{Su}$ rintendant des finances seit 1653, ein größeres Vermögen und Güter erwerben; Daniel DESSERT, Fortune politique et Politique de la fortune: à propos de la succession du Surintendant Abel Servien, in: La France d'Ancien Régime. Etudes réunies en l'honneur de Pierre Goubert, Toulouse 1984, Bd. 1, S. 207-214.

${ }^{68}$ F. UZuREAU, Abel Servien en Anjou, in: L'Anjou historique 23 (1923) S. 195-199, hier S. 197. Zur Ehe Serviens und dem biographischen Hintergrund seiner Frau siehe Jacques ISOLLE, Deux Angevins: Abel Servien, Augustine Le Roux et les traités de Westphalie. In: La Province du Maine (1977) S. 399-416. Erst nach dem Tod seiner Frau erwarb Servien den Titel eines Marquis von Sablé.

${ }^{69}$ Kettering (wie Anm. 11) S. 164; Kerviler (wie Anm. 13) S. 15f.; IsOlle (wie Anm. 68) S. 4f.

${ }^{70}$ RanUm (wie Anm. 9) S. 100; MoOTE (wie Anm. 43) S. 37. Als Servien seine politische Karriere fortsetzte, bemühte er sich, ein eigenes Verschulden an der Angelegenheit zurückzuweisen; siehe dazu seine Briefe an Lionne vom 3. September 1644 (APW II B 1 [wie Anm. 3] nr. 238 S. 496) und vom 27. Oktober 1648 (Konzept: AE, CP Allemagne 122 fol. 598-598').

${ }^{71}$ Georges DETHAN, Mazarin, un homme de paix à l'âge baroque 1602-1661, Paris 1981, S. $110 \mathrm{ff}$.

${ }^{72}$ Servien formulierte seine rhetorische Unterwerfung und Protektions-Bitten in Briefen an Mazarin und, gemäß der Natur dieser Korrespondenz, auch in solchen an Lionne; Beispiele finden sich in AE, CP Allemagne 38 fol. 76-78'; AE, CP Allemagne 78 fol. 411-413, 419-421, 422-424'; AE, CP Allemagne 119 fol. 46-46'. 
weil er außer Loyalität kaum etwas bieten konnte. Es war alles andere als zwangsläufig, daß Servien, der loyale Klient Mazarins, seinen Kollegen d'Avaux verdrängte. D'Avaux wurde als Person von eigenem politischem Gewicht von Mazarin in persönlichen Schreiben offen umworben ${ }^{73}$. Und der Kardinal hatte berechtigterweise lange eine hohe Meinung von d'Avaux' diplomatischer Kompetenz ${ }^{74}$. Dieser blieb jedoch politisch eigenständig. Diese Eigenständigkeit schlug mehr und mehr in Kritik um, die d'Avaux gemäßigt gegenüber Mazarin, jedoch schärfer auf dem Kongreß äußerte ${ }^{75}$. D'Avaux durchschaute Mazarins innenpolitische Notwendigkeiten. Gegenüber dem kaiserlichen Botschafter Trauttmansdorff erklärte er im Sommer 1646, es sei Mazarins vordringlichstes Ziel, möglichst viele eigene Klienten in gehobene Positionen zu bringen, und dabei schrecke er nicht einmal davor zurück, mißliebige Adelige im Krieg zu verheizen ${ }^{76}$. Es gab Gerüchte, d'Avaux strebe selbst nach der Regierung ${ }^{77}$. Wenn er solche Ambitionen hatte, so hat er sie niemals geäußert, doch entscheidend war, daß Mazarin diesen Gerüchten schließlich glaub$\mathrm{te}^{78}$.

Je mehr sich d'Avaux Mazarin entzog, desto mehr verschaffte er Servien einen Vorteil. Dieser attackierte schon während der gemeinsamen Reise im Dezember 1643 eine vermeintliche Führung, die d'Avaux aus seiner besseren Position heraus in der Gesandtschaft beanspruche ${ }^{79}$. Später eskalierte der Streit zwischen den Botschaftern, der oft darauf zurückgeführt wird, d'Avaux sei ein katholischer Religionspolitiker gewesen, Servien ein Vertreter der Staatsräson.

${ }^{73}$ Siehe dazu insbesondere Mazarins Briefe an d'Avaux vom 20. Juli und vom 10. August 1646; APW II B 4 (wie Anm. 19) nr. 80 und 108.

${ }^{74} \mathrm{Zu}$ Beginn des Kongresses betonte Mazarin einen besonderen Handlungsspielraum d'Avaux', entsprechend l'intelligence que vous avez des affaires et des mours des gens de ce pays là; APW II B 1 (wie Anm. 3) nr. 80 S. 158. Im Sommer 1646, als ein neuer Krieg zwischen Schweden und Polen auszubrechen drohte, forderte Mazarin insbesondere den Rat d'Avaux' an, der den letzten Waffenstillstand zwischen beiden Mächten vermittelt hatte; APW II B 4 (wie Anm. 19) nr. 4. Noch im April 1647 vertraute der Kardinal d'Avaux genügend, um ihm gegenüber Longuevilles Verhandlungsführung zu kritisieren; Ausfertigung: AE, CP Allemagne 99 fol. 288-290.

${ }^{75}$ D'Avaux' Kritik drang über Servien, aber auch über andere Quellen zu Mazarin. La Barde bestätigte Mazarin am 4. November 1645 entsprechende Aussagen Oxenstiernas; Ausfertigung: AE, CP Allemagne 45 fol. 107-110. Lionne referierte Vorwürfe gegen d'Avaux in Briefen an Servien vom 8. November 1647 und vom 14. Februar 1648; Ausfertigung: AE, CP Allemagne 103 fol. 119-120'; AE, CP Allemagne 118 fol. 359-361'. Vgl. auch Anm. 19.

${ }^{76}$ Trauttmansdorff berichtete am 3. Juli 1646 Ferdinand III. von diesem Gespräch; APW (wie Anm. 3) II A, Bd. 4, bearb. v. Hubert SALM und Brigitte WÜBBEKE-PFLÜGER, Münster 2001, nr. 234.

${ }^{77}$ Davon berichteten allerdings meistens Servien oder Lionne. Zu Prognosen bereits Ende der 30er Jahre, d'Avaux könne einmal die Regierungsführung übernehmen, siehe BOPPE (wie Anm. 13) S. 146.

${ }^{78}$ Lionne an Servien, 1648 April 17; Ausfertigung: AE, CP Allemagne 119 fol. 313.

${ }^{79}$ IRSIGLER, Einleitung zu APW II B 1 (wie Anm. 3) S. LXX. 
Dieses pauschale Urteil wird beiden nicht gerecht: Während auch Servien religionspolitische Ziele formulierte ${ }^{80}$, hatte d'Avaux Richelieus Politik im Reich und gegenüber Schweden, die man kaum als Religionspolitik bezeichnen kann, diplomatisch umgesetzt. Es gab zahlreiche, sehr vielschichtige Gegensätze zwischen den beiden Botschaftern, aber entscheidend ist, daß sie schon stritten, als von Inhalten noch gar nicht die Rede war. Die Inhalte, über die sie im Verlauf des Jahres 1644 stritten, waren - und so wurden sie vom Hof beurteilt $^{81}$ - Kleinigkeiten. Der Streit war in seinem Ursprung ein Führungsstreit in der Gesandtschaft, der daraus resultierte, daß Servien neu in ein Gebiet vordrang, in dem d'Avaux sich über Jahre hinweg eine feste Position geschaffen hatte.

Servien konnte dabei zunächst nicht auf Mazarins Unterstützung bauen. Mazarin nahm Servien gegenüber d'Avaux nicht in Schutz und forderte seinen Klienten mehrfach auf, Streit zu vermeiden ${ }^{82}$. Servien konnte sich aber auf seinen Neffen und politischen Zögling Lionne stützen, der seinen Onkel sogar rügte, wenn er sich gegenüber d'Avaux nachgiebig zeigte ${ }^{83}$. Lionne kontrollierte die Kanzlei Mazarins und, dank dessen Protektion, seit Juli 1646 auch die der Königin Anna ${ }^{84}$. Er besaß ein Informations-Monopol, das er völlig in

${ }^{80}$ So in Briefen an Lionne vom 10. Juni 1645 (APW II B 2 [wie Anm. 5] nr. 127 S. 417) und vom [14. Mai 1646] (APW II B 3 [wie Anm. 13] nr. 270) sowie an Longueville vom 18. April 1647 (Duplikat für Mazarin: AE, CP Hollande 44 fol. 126-135).

${ }^{81}$ Ludwig XIV. an d'Avaux und Servien, Paris 1645 Januar 1; APW II B 2 (wie Anm. 5) nr. 1 S. 6.

82 So durch Schreiben Lionnes vom 23. Juli 1644 (Beilage zu APW II B 1 [wie Anm. 3] nr. 181 S. 389) und vom 6. Mai 1645 (APW II B 2 [wie Anm. 5] nr. 98 S. $316 f$.). Lionne wies seinen Onkel bei diesen Gelegenheiten darauf hin, daß Mazarin beiden Botschaftern die Schuld an dem Streit gebe und keineswegs zugunsten Serviens voreingenommen sei. Später wies Mazarin Servien durch Lionne ebenfalls an, keinen Streit mit Longueville zu riskieren; APW II B 3 (wie Anm. 13) nr. 235; APW II B 4 (wie Anm. 19) nr. 56. Lionne warnte Servien in den ersten Jahren auch vor Alleingängen oder privaten Memoranden, deren Tenor zu stark von dem der gemeinsamen Depeschen differiere, da dies bei Mazarin keinen guten Eindruck hinterlasse; Lionne an Servien, Paris 1645 Januar 5; APW II B $2 \mathrm{nr} .5 \mathrm{~S} .21 \mathrm{f}$.

${ }^{83}$ Lionne an Servien, Paris 1645 Februar 4; ibid. nr. 33 S. 116f;; ders. an dens., Paris 1646 Februar 16; APW II B 3 (wie Anm. 13) nr. 119. In beiden Fällen kritisierte Lionne, daß sein Onkel Sonder-Missionen Saint-Romains nicht verhindert habe.

${ }^{84} \mathrm{Im}$ August 1646 gelang es Lionne mit Mazarins Unterstützung, die Nachfolge des verstorbenen Secrétaire des commandements de la Reine zu übernehmen, und im folgenden Jahr, eigene Sekretäre in der Kanzlei der Königin zu positionieren; Jérôme CRAS, Hugues de Lionne (1611-1671) (Thèse présentée pour l'obtention du diplôme d'archivistepaléographe), École nationale des chartes Paris 1995 (masch.), S. 98-106; APW II B 4 (wie Anm. 19) nr. 89, 97, 118, 128. Durch sein neues Amt kam Lionne zudem eine Schlüsselposition bei der Ernennung von Bischöfen und damit beim Aufbau Mazarins episkopaler Klientel zu; BERGIN (wie Anm. 11) S. 509f. 
den Dienst Serviens stellte ${ }^{85}$ : Er konnte Briefe Serviens aus der privaten Korrespondenz je nach Lage der Dinge vorlegen oder zurückhalten ${ }^{86}$. Er kannte fast alle Briefe Longuevilles und d'Avaux' an Mazarin ${ }^{87}$ und erfuhr frühzeitig von allen Entwicklungen und Regierungsentscheidungen, und er berichtete Servien darüber ${ }^{88}$. Als er im Frühjahr 1646 die Information erhielt, daß der zwischen den Botschaftern neutrale französische Resident in Osnabrück, La Barde, abberufen werden wollte, setzte Lionne in engem Kontakt mit Servien einen gemeinsamen Verwandten, Henri Groulart de La Court, als Nachfolger durch. D'Avaux erfuhr - wie bei der Abberufung Rortés 1645 - erst von den vollendeten Tatsachen, obwohl er lieber einen der ihm gewogenen Diplomaten als Nachfolger vorgeschlagen hätte ${ }^{89}$.

Diese Besetzung eines wichtigen diplomatischen Postens war ein Resultat von Beziehungen, keine Kompetenz-Entscheidung: D'Avaux' Klienten arbeiteten seit Jahren im Reich und wären kompetenter gewesen als der Neuling La Court. Servien aber gewann einen wichtigen Vorteil, denn La Court verstand sich als unbedingter Klient Serviens, dem er sich politisch unterordnete ${ }^{90}$. Servien kontrollierte nun besser als d'Avaux die Osnabrücker Verhandlungen und hatte einen Berichterstatter am Kongreß auf seiner Seite. 1647 konnte er sogar für ein halbes Jahr zu Verhandlungen nach Den Haag reisen und blieb doch über jeden Schritt d'Avaux', der in Osnabrück verhandelte, informiert.

Mazarin bildete sich ohnehin seine Meinung immer stärker anhand der Memoranden Serviens. Durch Longuevilles Anwesenheit blieb aber auf dem

${ }^{85}$ Memorandum Lionnes für Servien, 1644 Juni 25; APW II B 1 (wie Anm. 3) nr. 149 S. 288.

${ }^{86}$ Siehe dazu ein Schreiben Lionnes an Servien vom 5. Januar 1645; APW Il B 2 (wie Anm. 5) nr. 5 S. $21 \mathrm{f}$.

${ }^{87}$ D'Avaux bat Mazarin mehrmals vergeblich um Geheimhaltung, so in einem Brief vom 13. August (APW II B 4 [wie Anm. 19] nr. 113) und einem weiteren vom 24. Dezember 1646 (Ausfertigung: AE, CP Allemagne 80 fol. 117-119), in welchem er ausdrücklich bemerkte, Servien erhalte von allem Kenntnis, was Mazarin zu seinen offiziellen Papieren

- also in die Hand Lionnes - gebe. Am 6. März 1648 kündigte Lionne Servien sogar an, ihm ein Schreiben d'Avaux' an Mazarin zu kopieren und zu schicken; Ausfertigung: AE, CP Allemagne 119 fol. 86-87.

${ }^{88}$ Servien wurde von der Entfernung d'Avaux' und Saint-Romains vom Kongreß bereits vorab am 6. März 1648 durch Lionne informiert; Ausfertigung: AE, CP Allemagne 119 fol. 88-88'. D'Avaux hingegen wurde der Entschluß, ihn abzuberufen, erst am 13. März mitgeteilt (Ausfertigung: AE, CP Allemagne 79 fol. 305-305'), Saint-Romain seine Abberufung sogar erst am 20. März durch ein Schrẹiben Briennes an d'Avaux (Ausfertigung: ibid. fol. 308-308').

${ }^{89}$ Siehe dazu aus der Korrespondenz zwischen Servien und Lionne APW II B 3 (wie Anm. 13) nr. 256, 292; APW II B 4 (wie Anm. 19) nr. 5, 56; sowie: Servien an La Court, [Münster] 1646 Juli [7]; ibid. nr. 58.

${ }^{90} \mathrm{La}$ Court an Servien, Paris 1646 Juni 29; APW II B 4 (wie Anm. 19) nr. 39; ders. an dens., Osnabrück 1648 März 5; Ausfertigung: AE, CP Allemagne 107 fol. 296-297. 
Kongreß ein gewisser Ausgleich gewahrt. Als dieser im Februar 1648 abreiste, wurde auch bald darauf d'Avaux abberufen ${ }^{91}$. In gewisser Weise war dies eine Folge der Abreise Longuevilles, denn so zerstritten wie d'Avaux und Servien waren, konnte man sie nicht ohne den Herzog gemeinsam am Kongreß lassen. Servien hatte in den Jahren zuvor für sich, d'Avaux aber mit seiner Kritik gegen sich gearbeitet. Er brachte sich sogar mehrmals in den Verdacht, Instruktionen überschritten zu haben ${ }^{92}$. Im Dezember 1647 soll er dabei einer TeilRestitution Karls von Lothringen zugestimmt haben ${ }^{93}$. Es gibt für ein konkretes Fehlverhalten d'Avaux' keinen definitiven Beweis. Nach Aussagen Lionnes glaubte Mazarin den Gerüchten, wagte es wegen d'Avaux' starkem Rückhalt aber nicht, ihn ohne weiteres abzuberufen. Mazarin soll Lionne sogar aufgefordert haben, Beweise gegen d'Avaux zu fälschen ${ }^{94}$. Aber auch Longue-

91 Zur Situation nach der Abberufung d'Avaux' und zum Zusammenhang der französischen Kongreßpolitik, insbesondere gegenüber Spanien, mit der beginnenden Fronde siehe BÉLY (wie Anm. 19).

${ }^{92}$ Während eines Gesprächs mit Trauttmansdorff am 29. Juli 1646 überschritt d'Avaux wahrscheinlich seine Instruktionen, indem er den Spaniern in Aussicht stellte, Frankreich werde auf die Unterstützung der Aufstände in Portugal und Katalonien gegen Gebietsabtretungen in den Spanischen Niederlanden verzichten. Zu den näheren Umständen des Gesprächs und der Quellenlage siehe TISCHER, Einleitung zu APW II B 4 (wie Anm. 19).

${ }^{93}$ Zum Ursprung der politischen Problematik um Karl von Lothringen siehe Rainer BABEL, Zwischen Habsburg und Bourbon. Außenpolitik und europäische Stellung Herzog Karls IV. von Lothringen und Bar vom Regierungsantritt bis zum Exil (1624-1634) (Beihefte der Francia, 18), Sigmaringen 1989. Lothringen und Bar waren seit 1634 von Frankreich besetzt. Eine Zulassung des Herzogs zum Kongreß hatte d'Avaux selbst in den Präliminarverhandlungen 1641 verweigert. Ein Vermittlungsvorschlag des niederländischen Botschafters Knuyt von Ende Dezember 1647 zu sechs zwischen Frankreich und Spanien noch umstrittenen Verhandlungspunkten (Kopie einer französischen Zusammenfassung vom 1. Januar 1648: AE, CP Allemagne 107 fol. 2-2') sah die Restitution eines Kerngebiets, des ursprünglichen Herzogtums Lothringen, vor. Aus diesem Grund verweigerte die französische Delegation die Annahme des Vorschlags, mit dem im übrigen, wie die spätere Diskussion zeigte, keineswegs alle Probleme beseitigt gewesen wären. Umstritten ist, wie die französischen Botschafter im einzelnen auf den Vorschlag reagierten: D'Avaux plädierte in seinem Einzelschreiben (Kopie: AE, CP Allemagne 113 fol. 50-52) keineswegs für die Restitution. Nach der Schilderung Saint-Romains für Chavigny allerdings (Ausfertigung: AE, CP Allemagne 90 fol. 316-320') hätten Longueville und d'Avaux für die Annahme des Vermittlungsvorschlags gestimmt, doch habe Longueville die Zustimmung nach Einspruch Serviens zurückgezogen, so daß eine Nachfrage am Hof erfolgte. Eine königliche Instruktion vom 14. Oktober 1646 (APW II B 4 [wie Anm. 19] nr. 198) ermöglichte im übrigen eine lothringische Restitution unter zahlreichen Einschränkungen. Ihre tatsächliche Absicht, die in verschiedenen Schreiben erläutert wurde, war aber, Spanien eine Aufnahme seines Verbündeten, Herzog Karls, in den Vertrag zu ermöglichen, ohne ihn tatsächlich zu restituieren. So wurde diese Instruktion auch durch ein königliches Schreiben vom 17. Januar 1648 - als Reaktion auf Knuyts Vorschlag - erläutert; Kopie: AE, CP Allemagne 118 fol. 109-158'.

${ }^{94}$ Lionne an Servien, Paris 1648 Februar 7; Ausfertigung: AE, CP Allemagne 118 fol. 319. 
ville sprach nach seiner Rückkehr gegen d'Avaux ${ }^{95}$. Schließlich stimmte der Conseil, wo eine Mehrheit d'Avaux grundsätzlich gewogen war, seiner Abberufung $\mathrm{zu}^{96}$.

D'Avaux fiel zunächst in politische Ungnade und durfte seine Ämter nicht wahrnehmen ${ }^{97}$. Mazarin konnte mit d'Avaux aber nicht verfahren, wie es Richelieu mit Servien getan hatte, und d'Avaux erhielt seine Hofämter zurück ${ }^{98}$. An der ausbrechenden Fronde beteiligte d'Avaux sich übrigens nicht, sondern blieb bis zu seinem Tod im November 1650 loyal und stellte der Regierung sogar sein Privatvermögen zur Verfügung ${ }^{99}$.

Beziehungen, Patronage und das allgemeine politische Gewicht von Botschaftern spielten also in der französischen Diplomatie auf dem Westfälischen Friedenskongreß eine Rolle. Wesentliche Fragen dieser Diplomatie wurden aber erst während des Kongresses entschieden ${ }^{100}$. Die Botschafter wurden an diesen Entscheidungen ausdrücklich beteiligt ${ }^{101}$ und in einem gewissen Rahmen sogar

${ }^{95}$ Lionne an Servien, Paris 1648 März 6; Ausfertigung: AE, CP Allemagne 119 fol. 86-87. Vgl. auch SONNINo (wie Anm. 25) S. 231.

${ }^{96}$ Die Entlassung, die nicht mit d'Avaux' Verhalten sondern der spanischen Friedensunwilligkeit begründet wurde, teilte ein Schreiben Ludwigs XIV. d'Avaux am 13. März 1648 mit; Ausfertigung: AE, CP Allemagne 79 fol. 305-305'.

${ }^{97}$ Lionne an Servien, 1648 April 17; Ausfertigung: AE, CP Allemagne 119 fol. 313; ders. an dens., Paris 1648 Mai 15; Ausfertigung: AE, CP Allemagne 120 fol. 134-134'.

${ }^{98}$ Zur Rückkehr in den Conseil im Dezember 1648 siehe den Eintrag im Journal François Ogiers (2. Teil); BNF Fonds Français Nouvelles acquisitions 12845 fol. 21.1649 nahm er auch sein Amt als Surintendant des finances wieder wahr, legte es aber 1650 nieder; Richard BONNEY, Political Change in France under Richelieu and Mazarin 1624-1661, Oxford 1978, S. 61.

${ }^{99}$ Liste mit Anleihen der Krone, 1650 August 29: AE, MD France 871 fol. 421-423; Lionne an Gramont, 1650 September 8: Henri COURTEAULT (Hg.), Correspondance inédite du maréchal de Gramont et de Hugues de Lionne (Septembre-Décembre 1650), in: Annuaire-Bulletin de la Société de l'Histoire de France LXII (1925) S. 225-290, hier S. 235.

${ }^{100}$ Wenig erforscht ist die politische Entscheidungsfindung in anderen Delegationen. Wie Leopold Auer für die kaiserliche Kongreßpolitik bemerkt, fehlt einer solchen Untersuchung oft noch die Basis in Form von Quellenpublikationen zur Regierungstätigkeit und wissenschaftlichen Biographien der Entscheidungsträger; Leopold AUER, Die Ziele der kaiserlichen Politik bei den Westfälischen Friedensverhandlungen und ihre Umsetzung, in: Heinz DuchHARDT (Hg.), Der Westfälische Friede. Diplomatie - politische Zäsur kulturelles Umfeld - Rezeptionsgeschichte, München 1998, S. 143-173, hier S. $143 \mathrm{ff}$. Den Einfluß militärischer Entwicklungen auf die Kongreßpolitik untersucht CROXTON (wie Anm. 28).

${ }^{101}$ [...], il me semble bien juste de ne me déterminer qu'après que j'auray eu vos advis; Königin Anna an d'Avaux und Servien, Paris 1644 April 16; APW II B 1 (wie Anm. 3) nr. 45 S. 83. - [...], si ce n'est qu'on juge que l'advantage du service du Roy y [d. h. in der Instruktion; A.T.] demande quelque changement, dont vous donnerez s'il vous plaist advis par deçà et de vos sentiments que Sa Majesté fera examiner dans son Conseil et vous en envoyera ses ordres; Mazarin an dies., Paris 1644 April 16; ibid. nr. 46 S. 86. - Für seine Den Haag-Mission 1647 konzipierte Servien sogar seine eigene Instruktion, die man 
zum Handeln ohne Rückversicherung aufgefordert ${ }^{102}$. Mazarin machte Vorgaben, erwartete aber Selbständigkeit in Detailfragen, auch in solchen von weitreichender Konsequenz. So wollte er die Frage, ob man die Neuerwerbungen im Elsaß zu Lehen oder zu souveränem Besitz nehmen solle, von den Botschaftern entschieden sehen ${ }^{103}$. Auch in der Lothringen-Frage, die d'Avaux schließlich zum Verhängnis wurde, wurden die Botschafter immer wieder zu Stellungnahmen aufgefordert, und es war zumindest der Eindruck erweckt worden, selbst bisher ausgeschlossene Optionen seien offen ${ }^{104}$.

$\mathrm{Da}$ zwei gegensätzliche und zerstrittene Botschafter die Kongreßpolitik beeinflußten, wurde jedes Für und Wider jeweils sorgfältig erwogen und vielleicht verhindert, daß diese Politik starr in eine Richtung führte. Es gab grundlegende Interessen, in denen sich alle drei Botschafter nicht auseinanderdividieren ließen. Aber es gab auch Unterschiede zwischen d'Avaux und Servien.

Generell war d'Avaux gegenüber den Kriegsgegnern kompromißbereiter, Servien härter. Nach d'Avaux' Abberufung wurden prompt Vorwürfe laut, Mazarin sei friedensunwillig und habe deshalb den friedenswilligen d'Avaux abberufen ${ }^{105}$. Servien nahm - wie Mazarin selbst - alle künftigen Möglichkeiten in den Blick. Von der Richelieu'schen Protektions- und Passagen-Politik ${ }^{106}$

am Hof nicht einmal ausfertigte; dazu Schreiben Serviens an Lionne vom 24. Dezember 1646 (Konzept: AE, CP Allemagne 78 fol. 621-629') und Ludwigs XIV. an Servien vom 11. Januar 1647 (Ausfertigung: AE, CP Hollande 40 fol. 37-38').

${ }^{102}$ So in einem Brief Mazarins vom 3. Januar 1645: [...] nonobstant tous les ordres qu'on vous envoye Sa Majesté se remet à vous gouverner selon les conjunctures; APW II B 2 (wie Anm. 5) nr. 2 S. 15.

${ }^{103}$ Nachdem im Mai 1646 der kaiserliche Botschafter Trauttmansdorff angeboten hatte, Frankreich den abzutretenden Besitz im Elsaß nicht zu Lehen, sondern zu souveränem Besitz zu überlassen, bat die französische Regierung ihre Botschafter am 22. Juni um eine Stellungnahme (APW II B 4 [wie Anm. 19] nr. 23). Wegen unterschiedlicher Tendenzen in dieser Frage wurde ein ausgewogenes Gutachten erstellt, das keine eindeutige Empfehlung enthielt. Gerade dazu aber forderte Mazarin d'Avaux anschließend auf (ibid. nr. 146), weil auch er sich zu keinem Entschluß durchringen konnte. Eine Weile zeigte er sich von d'Avaux' Argumentation zugunsten der Lehensnahme überzeugt (ibid. nr. 200) und forderte ihn auf, ein entsprechendes gemeinsames Gutachten durchzusetzen. Dies unterblieb ebenso wie ein Regierungsbeschluß.

${ }^{104}$ Siehe dazu Schreiben Briennes und Mazarins vom 10. Februar bzw. vom 10. August 1646; APW II B 3 (wie Anm. 13) nr. 108; APW II B 4 (wie Anm. 19) nr. 108.

${ }^{105}$ Dazu die handschriftliche Kopie einer entsprechenden Flugschrift in AE, CP Allemagne 113 fol. 316-318'. In einer Parlamentsdebatte vom 17. Dezember 1648 (wie Anm. 65) wurde der Vorwurf laut, Longueville und d'Avaux seien abberufen worden, weil sie den Frieden zu weit voran getrieben hätten. Dem widersprachen allerdings sofort Condé und Parlamentspräsident Molé. Mazarin hatte solche Anschuldigungen bereits vorausgesehen, wie aus einem Schreiben Lionnes an Servien vom 3. Januar 1648 hervorgeht; Ausfertigung: AE, CP Allemagne 118 fol. 32-33.

${ }^{106}$ Richelieu hatte die Grundsätze dieser Politik nach der Einnahme La Rochelles und der Überwindung der innenpolitischen Probleme in einem Advis vom 13. Januar 1629 formuliert; Les Papiers de Richelieu, Section Politique Intérieure, Correspondance et Papiers 
hielt er nichts, sondern setzte auf Territorialerwerb ${ }^{107}$. D'Avaux wäre dagegen noch nach der Regelung der Satisfaktionsfrage im September $1646^{108}$ bereit gewesen, auf die elsässischen Neuerwerbungen zu verzichten zugunsten eines Kompromisses, der Frankreich Lothringen vertraglich sichern sollte ${ }^{109}$. Diese Diskussion wies Mazarin allerdings energisch zurück ${ }^{110}$. D'Avaux warnte Mazarin zudem vor weiteren Eroberungen oder Grenzverschiebungen ${ }^{111}$. In der Bündnispolitik wünschte d'Avaux eine größere Eigenständigkeit Frankreichs gegenüber den protestantischen Verbündeten bis hin zur Einmischung in konfessionelle und politische Belange, ohne das Bündnis selbst jemals in Frage zu stellen, und er konnte sich darauf berufen, daß dies unter Ludwig XIII. und Richelieu durchaus üblich gewesen $\operatorname{sei}^{112}$. Die politischen Gegensätze in

d'État, Bd. IV, bearb. v. Pierre GriLlon, Paris 1980, nr. 11, hier S. 25f. Zu Theorie und Praxis dieser Politik siehe Wolfgang Hans STEIN, Protection Royale. Eine Untersuchung zu den Protektionsverhältnissen im Elsaß zur Zeit Richelieus 1622-1643 (Schriftenreihe der Vereinigung zur Erforschung der Neueren Geschichte e.V., 9), Münster 1978; Hermann WEBER, Frankreich, Kurtrier, der Rhein und das Reich 1623-1635 (Pariser Historische Studien, 9), Bonn 1969.

${ }^{107}$ Seine Ablehnung von Protektionsrechten zugunsten souveräner Herrschaft formulierte Servien bereits in einem Brief an Lionne vom 25. Februar 1646; APW II B 3 (wie Anm. 13) nr. 136. Während der Diskussion um den Abtretungsmodus für das Elsaß favorisierte er die Souveränität und wollte diese vom Kaiser sogar über die dortigen Reichsstädte fordem, denn le simple droit de protection [...] est plus onéreux que profitable; APW II B 4 (wie Anm. 19) nr. 85. Als er sich mit dieser Forderung schon bei seinen Kollegen nicht durchsetzen konnte, machte er Lionne gegenüber keinen Hehl daraus, wie das mit der Landvogtei Hagenau zugestandene Protektionsrecht zu nutzen sei: [...] en usant prudemment et habilement du droict de protection qui nous y est acquis, on pourra establir avec le temps l'authorité du Roy dans lesdites villes tout de mesme que s'il en estoit le souverain absolu, car il me semble qu'il fault avoir pour but d'empescher que l'authorité de l'Empereur ne soit plus recognue à l'avenir dans tous les pays qui nous ont esté laissez au delà du Rhin; ibid. nr. 161.

${ }^{108}$ Konrad REPGEN, Die kaiserlich-französischen Satisfaktionsartikel vom 13. September 1646 - ein befristetes Agreement, in: Heinz DuchHARDT (Hg.), Der Westfälische Friede. Diplomatie - politische Zäsur - kulturelles Umfeld - Rezeptionsgeschichte, München 1998, S. 175-216.

${ }^{109} \mathrm{Er}$ stellte sich damit hinter einen niederländischen Vorschlag, das Elsaß zu restituieren, um dafür Lothringen vertraglich einzubehalten. Herzog Karl sollte von den Habsburgern, im Elsaß oder anderweitig, entschädigt werden; siehe dazu d'Avaux' Briefe an Mazarin vom 8. und 15. Oktober 1646; APW II B 4 (wie Anm. 19) nr. 190 und 204.

${ }^{110}$ Mazarin an d'Avaux, Paris 1646 Oktober 26; ibid. nr. 224.

"IIs ausreichende Grenzen Frankreichs nannte d'Avaux, der allerdings die Aussicht auf einen künftigen Erwerb der Spanischen Niederlande nie ablehnte, Breisach, Pinerolo und Perpignan. Eine Absage erteilte er Eroberungen in weiter entfernten Gebieten; APW II B 4 (wie Anm. 19) nr. 8 und 190.

${ }^{112}$ D'Avaux an Mazarin, Münster 1644 April 29; APW II B 1 (wie Anm. 3) nr. 76 S. 150ff.; ders. an Königin Anna, Münster 1644 April 29; ibid. nr. 77 S. 152f.; ders. an Mazarin, Münster 1646 April 30; APW II B 3 (wie Anm. 13) nr. 252; ders. an dens., Münster 1646 Mai 14; ibid. nr. 269; ders. an dens., Münster 1646 Juni 18; APW II B 4 (wie Anm. 19) nr. 19; ders. an dens., Münster 1646 August 13; ibid. nr. 113. Als d'Avaux 1647 in Osna- 
Schweden, die bis in die Osnabrücker Gesandtschaft hineinreichten, wollte d'Avaux ausnutzen, um den Verbündeten zur Mäßigung seiner Satisfaktionsforderungen zu zwingen ${ }^{113}$. Dagegen sah Servien Frankreichs Stärke in Abhängigkeit von seinem Bündissystem und warnte davor, dies zu gefährden ${ }^{114}$.

Wer seine jeweiligen Ansichten durchsetzen konnte, war grundsätzlich offen. Aus den oben dargelegten Gründen heraus wurde jedoch d'Avaux' Position immer schwächer, die Serviens stärker. So erreichte d'Avaux im Juni 1645 noch gegen den Widerstand Serviens und ohne Nachfrage beim Conseil, daß die zweite französische Proposition vom 11. Juni $1645^{115}$ nicht wortgleich mit der schwedischen abgefaßt wurde, sondern daß man religionspolitische Forderungen zugunsten der Protestanten auslie $\beta^{116}$. Tatsächlich hatten die Botschafter sich sogar selbst zunächst rückversichern wollen, weil man am Hof mit ihrer ersten Proposition vom 4. Dezember 1644 so unzufrieden gewesen war, daß eine revidierte Fassung herausgegeben werden mußte ${ }^{117}$. Dennoch wies die Regierung ihre Delegation in Münster im Vorfeld der zweiten Proposition an, verhandlungsverzögernde Nachfragen auch künftig zu unterlassen, so daß die Botschafter entscheidende Fragen notgedrungen untereinander entschieden $^{118}$.

brück ohne seine Kollegen verhandelte, nutzte er die Gelegenheit, Oxenstierna seine Einwände vorzutragen; Memorandum d'Avaux' für Ludwig XIV.; Ausfertigung: AE, CP Allemagne 82 fol. 219-227.

${ }^{113}$ D'Avaux an Mazarin, Münster 1646 Oktober 1; APW II B 4 (wie Anm. 19) nr. 182. Von den innerschwedischen Machtkämpfen war die französische Gesandtschaft auch durch Saint-Romain informiert, der im Juni von einer Gesandtschaftsreise aus Schweden nach Münster zurückgekehrt war.

${ }^{114}$ Memorandum Serviens für Lionne, [Münster] 1646 Oktober 3; ibid. nr. 183.

${ }^{115}$ Zum Zustandekommen der zweiten französischen Proposition siehe auch den Beitrag von Leopold Auer in diesem Band.

${ }^{116}$ Brienne hatte die Botschafter am 22. April 1645 angewiesen, keine religionspolitischen Forderungen zugunsten der Protestanten aufzunehmen, Lionne hatte dies gegenüber Servien am 29. bekräftigt; APW II B 2 (wie Anm. 5) nr. 86 S. 290, nr. 95 S. 310 . Damit war jedoch nicht die Frage thematisiert worden, ob gegebenenfalls die offene Abweichung von der schwedischen Proposition in Kauf zu nehmen sein, wofür d'Avaux erfolgreich plädierte. Servien hatte dagegen argumentiert, es sei ausreichend, deutlich zu machen, daß die religjonspolitischen Forderungen auf Schweden, nicht auf Frankreich zurückgingen. Die Auseinandersetzung schilderte d'Avaux in einem Schreiben an Brienne (Münster 1645 Mai 13; Ausfertigung: Assemblée Nationale Paris 274 fol. 516-519) und ein Memorandum Saint-Romains (APW II B 2 nr. 139 S. 436ff.). In ihrem gemeinsamen Bericht zur Proposition (ibid. nr. 143 S. 451 ff.) waren die Botschafter vorsichtig genug, sich lediglich auf die höfischen Anweisungen zu berufen und ihren daran anknüpfenden Streit nicht zu erwähnen.

${ }^{117}$ Die Anweisung, eine revidierte Fassung herauszugeben, erfolgte am 4. Januar 1645; ibid. nr. 3 S. 16ff.

${ }^{118}$ Memorandum Ludwigs XIV. für d'Avaux und Servien, Paris 1645 Mai 30; ibid. nr. 114 S. 385. Bereits die Instruktion zur Revision der ersten Proposition (s. o.) hatte die weiterhin bestehende Entscheidungsfreiheit der Botschafter betont, da Veränderungen nicht 
Anders war die Situation bereits im Oktober 1646, als d'Avaux auf eine Aufforderung des schwedischen Botschafters Salvius hin ${ }^{119}$ ein Schreiben der französischen Botschafter an Königin Christina anregte, um offiziell den hohen schwedischen Satisfaktionsforderungen entgegenzutreten. Longueville stimmte zu, aber Servien weigerte sich zu unterschreiben ${ }^{120}$. Dieses Vorgehen spricht bereits formal für sich, denn im Prinzip galt unter den Botschaftern der Mehrheitsentscheid ${ }^{121}$. Servien fühlte sich bereits so sicher, daß er keinen Mehrheitsbeschluß akzeptierte und sich dem Prinzipalbotschafter entgegensetzte. Er verweigerte auch die Diskussion und sandte seine Kritik an Lionne.

Bemerkenswert ist die Reaktion Mazarins: Am Hof erhielt man zunächst d'Avaux' Konzept für den Brief an Königin Christina ${ }^{122}$. Irrtümlich glaubte man, der Brief sei bereits abgeschickt worden, und er wurde gebilligt, auch von Mazarin ${ }^{123}$. Als Lionne den Kardinal aber von der Kritik Serviens informierte, zog dieser prompt seine Zustimmung zurück ${ }^{124}$. Der Brief blieb schließlich unausgefertigt.

Zusammenfassend läßt sich also sagen, daß personale Verflechtungen in der französischen Diplomatie auf dem Westfälischen Friedenskongreß in verschiedener Hinsicht eine Rolle spielten: Sie beeinflußten die Besetzung von Posten, aber auch die Frage, welcher der Botschafter sich bei Mazarin und der Regierung durchsetzte und welches Gewicht der einzelne in die Entscheidungen vor Ort, auf dem Kongreß, einbrachte. Damit beeinflußten sie also die politische Entscheidungsfindung sowie deren Umsetzung. Longuevilles und d'Avaux' größeren Klientelsystemen standen dabei Serviens wenige exklusive Beziehungen gegenüber. Ein maßgeblicher Faktor dieser personalen Verflechtungen war die politische Unterstützung, die aus ihnen resultierte. Ebenfalls

voraussehbar seien. Von der zweiten, in Münster umstrittenen Proposition nahm die französische Regierung später kaum Notiz.

${ }^{119}$ D'Avaux an Mazarin, Osnabrück 1646 September 23; APW II B 4 (wie Anm. 19) nr. 168. Servien bestätigte die Aufforderung durch Salvius in seinem Memorandum vom 9. Oktober (s. u.), bestritt aber, daß der Brief sich an Königin Christina habe richten sollen, und plädierte für ein Schreiben an den französischen Botschafter in Stockholm, Pierre Chanut, damit dieser persönlich auf die Königin einwirke.

${ }^{120}$ D'Avaux an Mazarin, Münster 1646 Oktober 8; ibid. nr 191; Memorandum Serviens für Lionne, Münster 1646 Oktober 9; ibid. nr. 192.

${ }^{121}$ Dieses Prinzip hatte d'Avaux bereits in einem Brief an Mazarin vom 29. April 1644 betont; APW II B 1 (wie Anm. 3) nr. $76 \mathrm{~S} .151$.

${ }^{122}$ Als Beilage 1 zu APW II B 4 (wie Anm. 19) nr. 182 vom 1. Oktober 1646.

${ }^{123}$ Brienne an Longueville, d'Avaux und Servien, Paris 1646 Oktober 12; ibid. nr. 193. Mazarin lobte das Schreiben an Königin Christina in einem Einzelschreiben an d'Avaux zwei Tage später: [...] la lettre que vous lwy avez escritte e[s]t trop persuasive pour n'en attendre pas au plus tost une response telle que l'on peut désirer. Je vous avoue cependant qu'on ne pouvoit escrire en termes plus pressans; ibid. nr. 200.

${ }^{124}$ Lionne an Servien, 1646 Oktober 19; Ausfertigung: AE, CP Allemagne 78 fol. 196-197. 
entscheidend war aber, daß gewisse Beziehungen einem Botschafter einen Informationsvorsprung verschaffen konnten, der es ihm zusätzlich ermöglichte, politisch und historisch entscheidende Vorgänge zu kontrollieren und zu beeinflussen. Auf dem Westfälischen Friedenskongreß war es Serviens Beziehung zu Lionne, die ihm diesen entscheidenden Vorsprung verschaffte, so daß er sich in einer vier Jahre dauernden Auseinandersetzung gegen d'Avaux' zunächst stärkere Beziehungen schließlich durchsetzte. 
